Homology, Homotopy and Applications, vol.3, No.1, 2001, pp.1-35

\title{
INTRODUCTION TO A-INFINITY ALGEBRAS AND MODULES
}

\author{
DEDICATED TO H. KELLER ON THE OCCASION OF HIS \\ SEVENTY FIFTH BIRTHDAY \\ BERNHARD KELLER \\ (communicated by Lionel Schwartz) \\ Abstract \\ These are expanded notes of four introductory talks on $A_{\infty}$ - \\ algebras, their modules and their derived categories.
}

\section{Contents}

1 Introduction 1

2 Motivation and topological origin 3

$3 A_{\infty}$-algebras and their morphisms $\quad 6$

$4 \begin{array}{ll}A_{\infty} \text {-modules and the derived category } & 14\end{array}$

$\begin{array}{lll}5 & \text { Triangulated structure } & 17\end{array}$

6 Standard functors 18

$\begin{array}{llr}7 & \text { Twisted objects } & 21\end{array}$

8 On the proofs $\quad 28$

\section{Introduction}

\subsection{These notes}

These are expanded notes of a minicourse of three lectures given at the Euroconference 'Homological Invariants in Representation Theory' in Ioannina, Greece, March 16 to 21, 1999, and of a talk at the Instituto de Matemáticas, UNAM, México, on April 28, 1999. They present basic results on $A_{\infty}$-algebras, their modules and their derived categories.

Partially supported by the European Network 'Algebraic Lie Representations', Contract ERBFMRX-CT97-0100

Received January 17, 2000, revised January 13, 2001; published on January 22, 2001.

2000 Mathematics Subject Classification: 18E30, 16D90, 18G40, 18G10, 55 U35

Key words and phrases: $A$-infinity algebra, Derived category

(C) 2001, Bernhard Keller. Permission to copy for private use granted. 


\subsection{History}

$A_{\infty}$-spaces and $A_{\infty}$-algebras (= sha algebras $=$ strongly homotopy associative algebras) were invented at the beginning of the sixties by J. Stasheff [45] [46] as a tool in the study of 'group-like' topological spaces. In the subsequent two decades, $A_{\infty}$-structures found applications and developments [30] [3] [1] in homotopy theory; their use remained essentially confined to this subject (cf. however [34], [42]). This changed at the beginning of the nineties when the relevance of $A_{\infty}$-structures in algebra, geometry and mathematical physics became more and more apparent (cf. e.g. [11], [48], [31]). Of special influence was M. Kontsevich's talk [23] at the International Congress in 1994: Inspired by K. Fukaya's preprint [8] Kontsevich gave a conjectural interpretation of mirror symmetry as the 'shadow' of an equivalence between two triangulated categories associated with $A_{\infty}$-categories. His conjecture was proved in the case of elliptic curves by A. Polishchuk and E. Zaslow [37].

\subsection{Scope and sources}

In these notes, we aim at presenting some basic theorems on $A_{\infty}$-algebras, their modules and their derived categories. The notes may serve as an introduction to a (very) small part of Kontsevich's lectures [25]. Besides these, our main sources were $[\mathbf{3 8}],[\mathbf{4 6}],[\mathbf{4 3}],[\mathbf{1 5}],[\mathbf{1 6}],[\mathbf{1 8}]$. Some of the results we state are not proved in this form in the literature. Complete proofs will be given in $[\mathbf{2 8}]$.

\subsection{Contents}

In section 2 , we motivate the introduction of $A_{\infty}$-algebras and modules by two basic problems from homological algebra:

1. the reconstruction of a complex from its homology,

2. the reconstruction of the category of iterated selfextensions of a module from its extension algebra.

Then we briefly present the topological origin of $A_{\infty}$-structures. Section 3 is devoted to $A_{\infty}$-algebras and their morphisms. The central result is the theorem on the existence of minimal models. In sections 4 and 5, we introduce the derived category of an $A_{\infty}$-algebra and we present the natural framework for the solution of problem 1. In section 6 , we sketch the formalism of standard functors and arrive at the solution of problem 2. Section 7 presents the category of twisted objects, which is of importance because it is 'computable'.

\subsection{Omissions}

The links with Morita theory for derived categories [39] or dg categories [21] have not been made explicit, cf. however [22].

The relevance of $A_{\infty}$-algebras to boxes and matrix problems was discovered by S. Ovsienko [34] (cf. also [35]). We only give a hint of this important development in example 7.9 .

The notions of $A_{\infty}$-equivalence and of $A_{\infty}$-enhanced triangulated categories, crucial for $[\mathbf{2 5}]$, will be treated in $[\mathbf{2 8}]$.

Acknowledgments. The author is grateful to N. Marmaridis and C. M. Ringel for 
the organization of the Euroconference in Ioannina, Greece, where the material of sections 2 to 6 was first presented. He expresses his sincere thanks to J. A. de la Peña and C. Geiss for the hospitality he enjoyed at the Instituto de Matemáticas, UNAM, México, where the material of section 7 was first presented and a first draft of these notes completed.

\section{Motivation and topological origin}

\subsection{Motivation}

Let $k$ be a field. The following two problems will guide us in our exploration of the world of $A_{\infty}$-algebras and modules.

Problem 1. Let $A$ be an associative $k$-algebra with 1 , let

$$
M=\left(\ldots \rightarrow M^{p} \stackrel{d^{p}}{\rightarrow} M^{p+1} \rightarrow \ldots\right)
$$

be a complex of (unital right) $A$-modules and let

$$
H^{*} M=\operatorname{ker} d^{*} / \operatorname{im} d^{*}, * \in \mathbf{Z},
$$

be its homology. It is a naive but natural question to ask whether, up to quasiisomorphism, we can reconstruct the complex $M$ from its homology $H^{*} M$. This is of course impossible, except under very restrictive hypotheses, for example when $A$ is an hereditary algebra (i.e. an algebra such that submodules of projective $A$ modules are projective, see [6]). So we ask what additional structure is needed if we want to reconstruct $M$ from its homology. The answer is that $H^{*} M$ carries a unique (up to isomorphism) $A_{\infty}$-module structure over $A$ (viewed as an $A_{\infty}$-algebra) which encodes exactly the additional information needed for this task.

Problem 2. Let $B$ be an associative $k$-algebra with 1 , let $M_{1}, \ldots, M_{n}$ be $B$ modules and let filt $\left(M_{i}\right)$ denote the full subcategory of the category of right $B$ modules whose objects admit finite filtrations with subquotients among the $M_{i}$. In other words, filt $\left(M_{i}\right)$ is the closure under extensions of the $M_{i}$. A natural question to ask is whether filt $\left(M_{i}\right)$ is determined by the extension algebra

$$
\operatorname{Ext}_{B}^{*}(M, M), \text { where } M=\bigoplus M_{i},
$$

together with its idempotents corresponding to the $M_{i}$. Again, the answer is no. This time, it is no even if we assume that $B$ is hereditary. Again, we ask what additional structure on the extension algebra is needed to reconstruct the category of iterated extensions. The answer is that the extension algebra admits an $A_{\infty}$-structure which encodes the additional information needed for this task.

The aim of these lectures is to explain the answers to the two problems and to show how they fit into a general theory.

\subsection{Topological origin}

At the beginning of the sixties, J. Stasheff invented $A_{\infty}$-spaces and $A_{\infty}$-algebras $[\mathbf{4 5}],[\mathbf{4 6}]$ as a tool in the study of 'group-like' spaces. Let us consider the basic example: 
Let $(X, *)$ be a topological space with a base point $*$ and let $\Omega X$ denote the space of based loops in $X$ : a point of $\Omega X$ is thus a continuous map $f: S^{1} \rightarrow X$ taking the base point of the circle to the base point $*$. We have the composition map

$$
m_{2}: \Omega X \times \Omega X \rightarrow \Omega X
$$

sending a pair of loops $\left(f_{1}, f_{2}\right)$ to the loop $f_{1} * f_{2}=m_{2}\left(f_{1}, f_{2}\right)$ obtained by running through $f_{1}$ on the first half of the circle and through $f_{2}$ on the second half

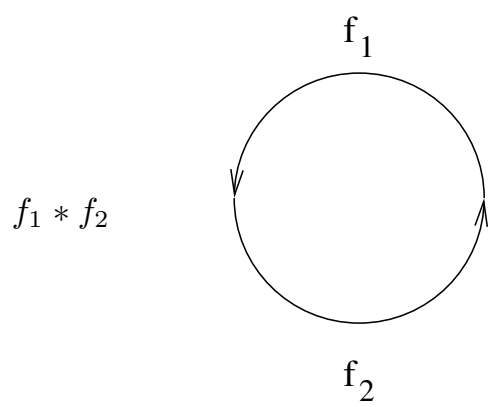

This composition is non associative: for three loops $f_{1}, f_{2}, f_{3}$, the composition $\left(f_{1} * f_{2}\right) * f_{3}$ runs through $f_{1}$ on the first quarter of the circle whereas the composition $f_{1} *\left(f_{2} * f_{3}\right)$ runs through $f_{1}$ on the first half of the circle. We symbolize the two possibilities by the two binary trees with three leaves

$\left(f_{1} * f_{2}\right) * f_{3}$
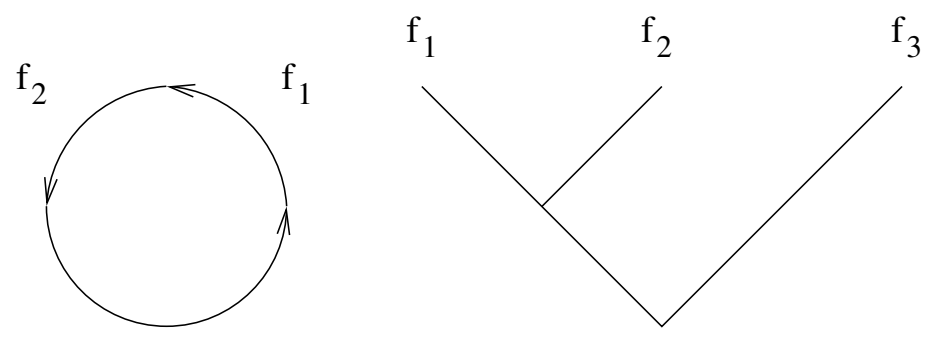

$\mathrm{f}_{3}$

$f_{1} *\left(f_{2} * f_{3}\right)$
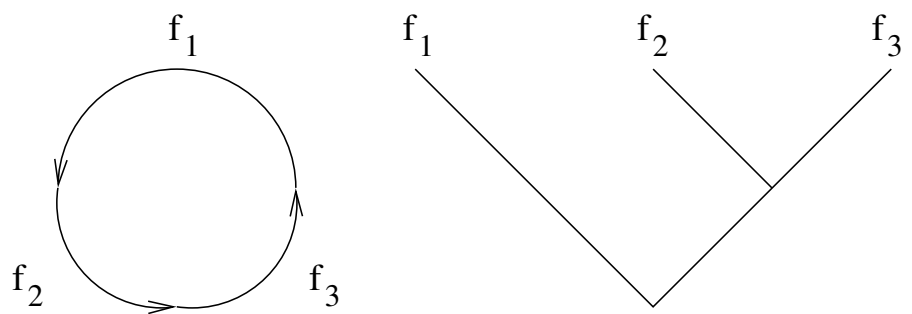
Clearly, there is an homotopy

$$
m_{3}:[0,1] \times \Omega X \times \Omega X \rightarrow \Omega X .
$$

joining the two possibilities of composing three loops. When we want to compose 4 factors, there are 5 possibilities corresponding to the 5 binary trees with 4 leaves. Using $m_{3}$, we obtain two paths of homotopies joining the composition $\left(f_{1}, f_{2}, f_{3}, f_{4}\right) \mapsto$ $\left(\left(f_{1} * f_{2}\right) * f_{3}\right) * f_{4}$ to the composition $\left(f_{1}, f_{2}, f_{3}, f_{4}\right) \mapsto f_{1} *\left(f_{2} *\left(f_{3} * f_{4}\right)\right)$.

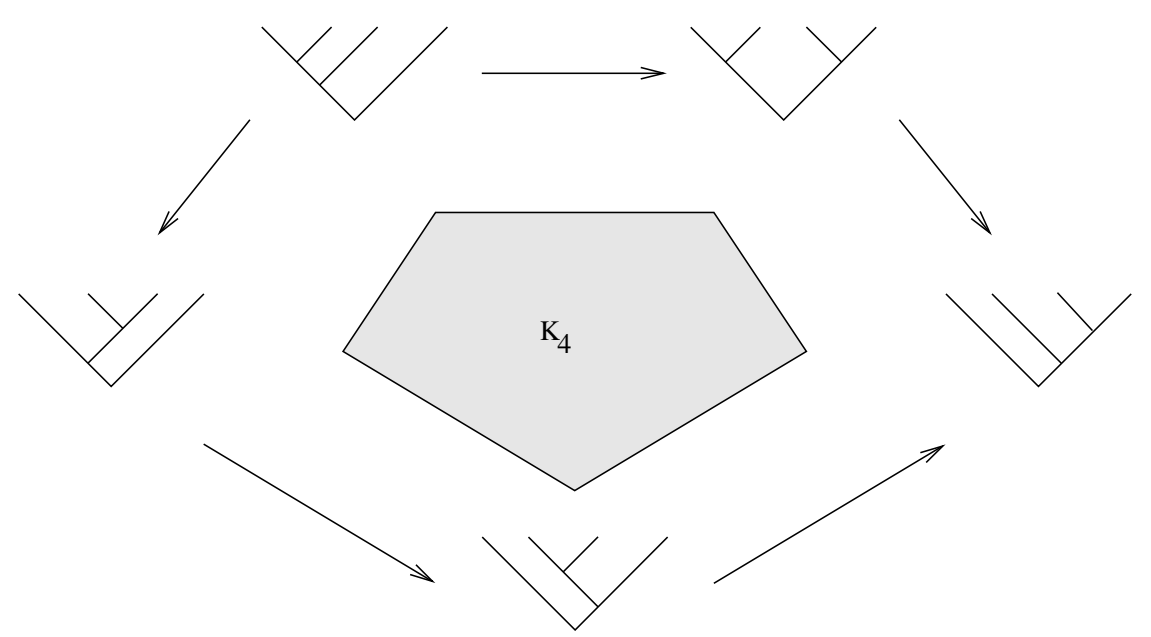

These two paths are clearly homotopic. Let us denote an homotopy by

$$
m_{4}: K_{4} \times(\Omega X)^{4} \rightarrow \Omega X
$$

where $K_{4}$ denotes the pentagon bounded by the two paths. When we want to compose 5 factors, there are 14 possibilities corresponding to the 14 binary trees with 5 leaves. Using $m_{4}$ and $m_{3}$, we obtain paths linking the compositions and faces linking the paths. The resulting sphere is the boundary of the polytope $K_{5}$ depicted 
[48] below.

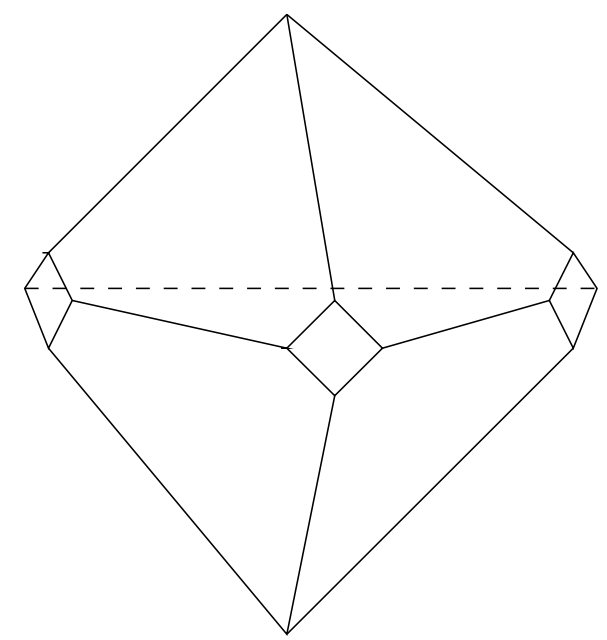

The pentagonal faces are copies of $K_{4}$.

More generally, Stasheff [45] defined polytopes $K_{n}$ of dimension $n-2$ for all $n \geqslant 2$ (we have $K_{2}=*, K_{3}=[0,1]$ ) and he defined an $A_{\infty}$-space to be a topological space $Y$ endowed with maps $m_{n}: K_{n} \times Y^{n} \rightarrow Y, n \geqslant 2$, satisfying suitable compatibility conditions and admitting a 'strict unit'. The loop space $\Omega X$ is the prime example of such a space $Y$. Conversely $[\mathbf{1}, 2.2]$, a topological space that admits the structure of an $A_{\infty}$-space and whose connected components form a group is homotopy equivalent to a loop space.

If $Y$ is an $A_{\infty}$-space, the singular chain complex of $Y$ is the paradigmatic example of an $A_{\infty}$-algebra $[46]$.

\section{3. $A_{\infty}$-algebras and their morphisms}

\subsection{Definition of an $A_{\infty}$-algebra}

Let $k$ be a field. An $A_{\infty}$-algebra over $k$ (also called a 'strongly homotopy associative algebra' or an 'sha algebra') is a $\mathbf{Z}$-graded vector space

$$
A=\bigoplus_{p \in \mathbf{Z}} A^{p}
$$

endowed with graded maps (=homogeneous $k$-linear maps)

$$
m_{n}: A^{\otimes n} \rightarrow A, n \geqslant 1,
$$

of degree $2-n$ satisfying the following relations

- We have $m_{1} m_{1}=0$, i.e. $\left(A, m_{1}\right)$ is a differential complex.

- We have

$$
m_{1} m_{2}=m_{2}\left(m_{1} \otimes \mathbf{1}+\mathbf{1} \otimes m_{1}\right)
$$

as maps $A^{\otimes 2} \rightarrow A$. Here $\mathbf{1}$ denotes the identity map of the space $A$. So $m_{1}$ is a (graded) derivation with respect to the multiplication $m_{2}$. 
- We have

$$
\begin{aligned}
m_{2}\left(\mathbf{1} \otimes m_{2}-\right. & \left.m_{2} \otimes \mathbf{1}\right) \\
& =m_{1} m_{3}+m_{3}\left(m_{1} \otimes \mathbf{1} \otimes \mathbf{1}+\mathbf{1} \otimes m_{1} \otimes \mathbf{1}+\mathbf{1} \otimes \mathbf{1} \otimes m_{1}\right)
\end{aligned}
$$

as maps $A^{\otimes 3} \rightarrow A$. Note that the left hand side is the associator for $m_{2}$ and that the right hand side may be viewed as the boundary of $m_{3}$ in the morphism complex $\operatorname{Hom}_{k}{ }_{k}\left(A^{\otimes 3}, A\right)$ (the definition of the morphism complex is recalled below). This implies that $m_{2}$ is associative up to homotopy.

- More generally, for $n \geqslant 1$, we have

$$
\sum(-1)^{r+s t} m_{u}\left(\mathbf{1}^{\otimes r} \otimes m_{s} \otimes \mathbf{1}^{\otimes t}\right)=0
$$

where the sum runs over all decompositions $n=r+s+t$ and we put $u=r+1+t$.

We have adopted the sign conventions of Getzler-Jones [11]. Note that when these formulas are applied to elements, additional signs appear because of the Koszul sign rule:

$$
(f \otimes g)(x \otimes y)=(-1)^{|g||x|} f(x) \otimes g(y)
$$

where $f, g$ are graded maps, $x, y$ homogeneous elements and the vertical bars denote the degree. For example, we have

$$
\left(m_{1} \otimes \mathbf{1}+\mathbf{1} \otimes m_{1}\right)(x \otimes y)=m_{1}(x) \otimes y+(-1)^{|x|} x \otimes m_{1}(y)
$$

so that $m_{1} \otimes \mathbf{1}+\mathbf{1} \otimes m_{1}$ is the usual differential on the tensor product. The defining identities are pictorially represented $[\mathbf{2 5}]$ by

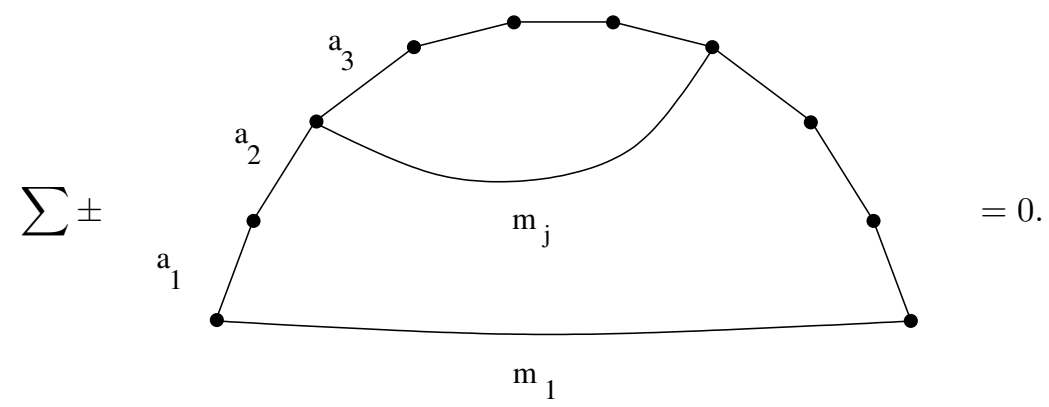

We note three immediate consequences of the definition:

(1) In general, an $A_{\infty}$-algebra $A$ is not associative but its homology $H^{*} A=$ $H^{*}\left(A, m_{1}\right)$ is an associative graded algebra for the multiplication induced by $m_{2}$.

(2) If $A^{p}=0$ for all $p \neq 0$, then $A=A^{0}$ is an ordinary associative algebra. Indeed, since $m_{n}$ is of degree $2-n$, all $m_{n}$ other than $m_{2}$ have to vanish.

(3) If $m_{n}$ vanishes for all $n \geqslant 3$, then $A$ is an associative $\operatorname{dg}$ (=differential $\mathbf{Z}$ graded) algebra and conversely each dg algebra yields an $A_{\infty}$-algebra with $m_{n}=0$ for all $n \geqslant 3$. 
Note that we have not defined unitality for $A_{\infty}$-algebras. Cf. however 3.5 below.

\subsection{Link with deformation theory}

The following class of examples, due to Penkava-Schwarz [36], shows the close link between deformation theory and $A_{\infty}$-algebras (cf. also [26]): Let $B$ be an ordinary associative algebra, $n \geqslant 1$ an integer and $\varepsilon$ an indeterminate of degree $2-n$ and with $\varepsilon^{2}=0$. An infinitesimal n-deformation of $B$ is a $k[\varepsilon]$-multilinear $A_{\infty}$-algebra structure on $B \otimes k[\varepsilon]$ which lifts the multiplication of $B$. Thus an infinitesimal 2 -deformation is just an algebra deformation of $B$. It is well-known and easy to check that infinitesimal 2-deformations are in natural bijection with Hochschild 2-

cocycles $c: B^{\otimes 2} \rightarrow B$. Analogously, infinitesimal $n$-deformations are in natural bijection with Hochschild $n$-cocycles. Thus, by working in the class of $A_{\infty}$-algebras, we may interpret Hochschild cocycles of arbitrary degree $\geqslant 1$ as deformations.

\subsection{Minimal models}

Let us recall the dg algebra which is implicit in problem 2: Let $B$ be an ordinary associative $k$-algebra with 1 and and $M$ a $B$-module. Choose an injective resolution

$$
0 \rightarrow M \rightarrow I^{0} \rightarrow I^{1} \rightarrow \ldots
$$

of $M$ and denote by $I$ the complex

$$
0 \rightarrow I^{0} \rightarrow I^{1} \rightarrow \ldots
$$

Let $\operatorname{Hom}_{B}{ }_{B}(I, I)$ be the morphism complex: recall that its $n$th component is the group of graded $B$-linear maps $f: I \rightarrow I$ of degree $n$ (no compatibility with $d_{I}$ ) and its differential is defined by $d(f)=d_{I} \circ f-(-1)^{n} f \circ d_{I}$. Then $A=\operatorname{Hom}_{B}(I, I)$ endowed with its differential becomes a dg algebra for the natural composition of graded maps and its homology algebra is the extension algebra of $M$ :

$$
H^{*} A=\operatorname{Ext}_{B}^{*}(M, M) .
$$

We claim that this has a canonical (up to equivalence) structure of $A_{\infty}$-algebra with $m_{1}=0$ and $m_{2}$ the usual multiplication. Indeed, more generally, we have the

Theorem (Kadeishvili [15], see also [14], [43], [38], [12], [13], [32]). Let $A$ be an $A_{\infty}$-algebra. Then the homology $H^{*} A$ has an $A_{\infty}$-algebra structure such that

1) we have $m_{1}=0$ and $m_{2}$ is induced by $m_{2}^{A}$ and

2) there is a quasi-isomorphism of $A_{\infty}$-algebras $H^{*} A \rightarrow A$ lifting the identity of $H^{*} A$.

Moreover, this structure is unique up to (non unique) isomorphism of $A_{\infty}$-algebras.

The notion of morphism and quasi-isomorphism of $A_{\infty}$-algebras will be defined below (3.4). The most explicit construction of the $A_{\infty}$-structure on $H^{*} A$ can be found in Merkulov's [32]. His construction is explained in terms of trees in $[\mathbf{2 7}, 6.4]$.

Let us introduce some terminology: An $A_{\infty}$-algebra with vanishing $m_{1}$ is called minimal. In the situation of the theorem, the $A_{\infty}$-algebra $H^{*} A$ is called a minimal model for $A$. The $A_{\infty}$-algebra $A$ is called formal if its minimal model can be chosen to be an ordinary associative graded algebra, i.e. such that $m_{n}=0$ for all $n \geqslant 3$. 
A graded algebra $B$ is intrinsically formal if each $A_{\infty}$-algebra $A$ whose homology is isomorphic to $B$ is formal. A sufficient condition for $B$ to be intrinsically formal in terms of its Hochschild homology is given in [19], cf. also [41, 4.7].

It was shown in $[\mathbf{7}]$ that the dg algebra of smooth real forms on a compact Kähler manifold is formal. In particular, this holds for smooth, complex projective manifolds.

In contrast to the statement of the above theorem, one can also show that each $A_{\infty}$-algebra $A$ admits an 'anti-minimal' model, i.e. there is a dg algebra $C$ and a quasi-isomorphism of $A_{\infty}$-algebras $A \rightarrow C$. Thus, passing from dg algebras to $A_{\infty^{-}}$ algebras does not yield new quasi-isomorphism classes. What it does yield is a new description of these classes by minimal models.

If, in the above example, we choose $B$ to be a finite-dimensional algebra and $M$ to be the sum of the simple $B$-modules, one can show that the $\operatorname{dg}$ algebra $A$ is formal iff $B$ is a Koszul algebra. The smallest examples where $A$ is not formal is the algebra $B$ defined by the quiver with relations (cf. $[\mathbf{4 0}],[\mathbf{9}],[\mathbf{2}]$ )

$$
\bullet \stackrel{\alpha}{\rightarrow} \bullet \stackrel{\beta}{\rightarrow} \bullet \stackrel{\gamma}{\rightarrow} \bullet, \gamma \beta \alpha=0
$$

Here the extension algebra $H^{*} A$ is given by the quiver with relations

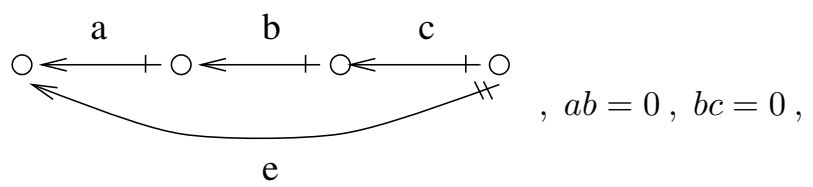

where the arrows $a, b, c$ are of degree 1 and the arrow $e$ is of degree 2 . We have $m_{n}=0$ for $n \neq 2,3, m_{3}(a, b, c)=e$ and all other products vanish (the method for computing this is given at the end of section 3.4). This example illustrates the fact that the higher products of the $A_{\infty}$-algebra $H^{*} A$ lift the Massey products [46] whenever these are defined.

\subsection{Morphisms of $A_{\infty}$-algebras}

A morphism of $A_{\infty}$-algebras $f: A \rightarrow B$ is a family

$$
f_{n}: A^{\otimes n} \rightarrow B
$$

of graded maps of degree $1-n$ such that

- we have $f_{1} m_{1}=m_{1} f_{1}$, i.e. $f_{1}$ is a morphism of complexes,

- we have

$$
f_{1} m_{2}=m_{2}\left(f_{1} \otimes f_{1}\right)+m_{1} f_{2}+f_{2}\left(m_{1} \otimes \mathbf{1}+\mathbf{1} \otimes m_{1}\right),
$$

which means that $f_{1}$ commutes with the multiplication $m_{2}$ up to a homotopy given by $f_{2}$,

- more generally, for $n \geqslant 1$, we have

$$
\sum(-1)^{r+s t} f_{u}\left(\mathbf{1}^{\otimes r} \otimes m_{s} \otimes \mathbf{1}^{\otimes t}\right)=\sum(-1)^{s} m_{r}\left(f_{i_{1}} \otimes f_{i_{2}} \otimes \ldots \otimes f_{i_{r}}\right)
$$

where the first sum runs over all decompositions $n=r+s+t$, we put $u=$ $r+1+t$, and the second sum runs over all $1 \leqslant r \leqslant n$ and all decompositions 


$$
\begin{aligned}
& n=i_{1}+\cdots+i_{r} \text {; the sign on the right hand side is given by } \\
& \qquad \quad s=(r-1)\left(i_{1}-1\right)+(r-2)\left(i_{2}-1\right)+\cdots+2\left(i_{r-2}-1\right)+\left(i_{r-1}-1\right) .
\end{aligned}
$$

The morphism $f$ is a quasi-isomorphism if $f_{1}$ is a quasi-isomorphism. It is strict if $f_{i}=0$ for all $i \neq 1$. The identity morphism is the strict morphism $f$ such that $f_{1}$ is the identity of $A$. The composition of two morphisms $f: B \rightarrow C$ and $g: A \rightarrow B$ is given by

$$
(f \circ g)_{n}=\sum(-1)^{s} f_{r} \circ\left(g_{i_{1}} \otimes \cdots \otimes g_{i_{r}}\right)
$$

where the sum and the sign are as in the defining identity. Here is a (slightly stylized) pictorial representation of the defining identity for a morphism of $A_{\infty}$-algebras
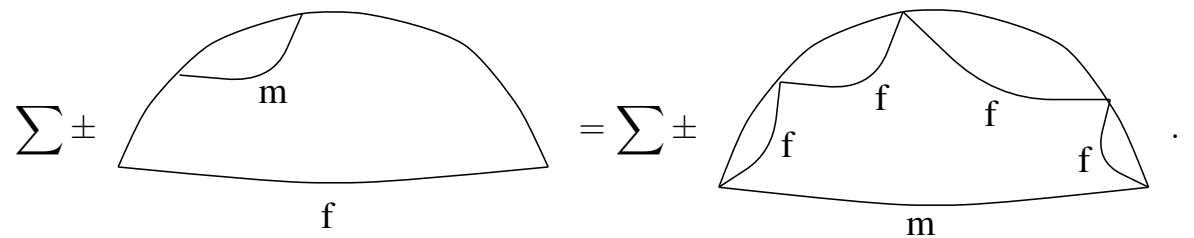

$A_{\infty}$-algebras may be viewed as algebras over an operad (cf. e.g. [29]). However, only strict morphisms of $A_{\infty}$-algebras may then be viewed as morphisms of algebras.

As an illustration of the notion of morphism, suppose that $A$ is a $\operatorname{dg}$ algebra and let us construct the multiplication $m_{3}$ on $H^{*} A$ and, at the same time, the first three terms of a quasi-isomorphism $f: H^{*} A \rightarrow A$ lifting the identity of $H^{*} A$. For this, we view $H^{*} A$ as a complex with zero differential and we choose a morphism of complexes $f_{1}: H^{*} A \rightarrow A$ inducing the identity in homology. Then $f_{1}$ commutes with the multiplication up to boundaries, i.e. there exists a graded map $f_{2}: H^{*} A \otimes$ $H^{*} A \rightarrow A$ degree -1 such that

$$
f_{1} m_{2}=m_{2}\left(f_{1} \otimes f_{1}\right)+m_{1} f_{2} .
$$

Now we look for $f_{3}$ and $m_{3}$ such that

$$
\begin{aligned}
& f_{1} \circ m_{3}+f_{2} \circ\left(m_{2} \otimes \mathbf{1}-\mathbf{1} \otimes m_{2}\right) \\
& +f_{3} \circ\left(m_{1} \otimes \mathbf{1}^{\otimes 2}+\mathbf{1} \otimes m_{1} \otimes \mathbf{1}+\mathbf{1}^{\otimes 2} \otimes m_{1}\right) \\
= & m_{3} \circ\left(f_{1} \otimes f_{1} \otimes f_{1}\right)+m_{2} \circ\left(f_{1} \otimes f_{2}-f_{2} \otimes f_{1}\right)+m_{1} f_{3} .
\end{aligned}
$$

In our situation, this simplifies into

$$
f_{1} \circ m_{3}=m_{2} \circ\left(f_{1} \otimes f_{2}-f_{2} \otimes f_{1}\right)+m_{1} f_{3} .
$$

Now it is easy to check that the map $m_{2}\left(f_{1} \otimes f_{2}-f_{2} \otimes f_{1}\right)$ has its image in the cycles of $A$. So there is indeed a map $f_{3}:\left(H^{*} A\right)^{\otimes 3} \rightarrow A$ of degree -2 and a map $m_{3}:\left(H^{*} A\right)^{\otimes 3} \rightarrow H^{*} A$ of degree -1 such that the identity holds.

\subsection{Augmented $A_{\infty}$-algebras}

If an $A_{\infty}$-algebra $A$ has a unital homology algebra $H^{*} A$, one can often simplify the computation of the minimal model for $A$ by passing to the category of augmented $A_{\infty}$-algebras. Here are the relevant definitions: 
A strictly unital $A_{\infty}$-algebra is an $A_{\infty}$-algebra $A$ endowed with an element $1_{A}$ of degree 0 such that we have $m_{1}\left(1_{A}\right)=0, m_{2}\left(1_{A}, a\right)=a=m_{2}\left(a, 1_{A}\right)$ for all $a \in A$ and such that, for all $i>2$, and all $a_{1}, \ldots, a_{i}$ in $A$, the product $m_{i}\left(a_{1}, \ldots, a_{i}\right)$ vanishes if one of the $a_{j}$ equals $1_{A}$. If $A$ and $B$ are strictly unital $A_{\infty}$-algebras, a morphism of $A_{\infty}$-algebras $f: A \rightarrow B$ is strictly unital if we have $f_{1}\left(1_{A}\right)=1_{B}$ and if for all $i>1$ and all $a_{1}, \ldots, a_{i}$, the element $f_{i}\left(a_{1}, \ldots, a_{i}\right)$ vanishes if one of the $a_{j}$ equals 1 . Each strictly unital $A_{\infty}$-algebra is canonically endowed with a strict (hence strictly unital) morphism $\eta: k \rightarrow A$ mapping $1_{k}$ to $1_{A}$. It is augmented if it is moreover endowed with a strictly unital morphism $\varepsilon: A \rightarrow k$ such that $\varepsilon \circ \eta=\mathbf{1}_{k}$. A morphism of augmented $A_{\infty}$-algebras is a strictly unital morphism $f: A \rightarrow B$ such that $\varepsilon_{B} \circ f=\varepsilon_{A}$. It is then easy to check that the functor $A \mapsto \operatorname{ker} \varepsilon$ is an equivalence from the category of augmented $A_{\infty}$-algebras to the category of $A_{\infty^{-}}$ algebras. The quasi-inverse functor sends an $A_{\infty}$-algebra $B$ to $k \oplus B$ endowed with the unique strictly unital structure extending the structure of $B$.

Let $A$ be an augmented $A_{\infty}$-algebra and let $\bar{A}=\operatorname{ker} \varepsilon$. Clearly we obtain a minimal model for $A$ by taking $k \oplus \bar{A}^{\prime}$, where $\bar{A}^{\prime}$ is a minimal model for $\bar{A}$ in the category of $A_{\infty}$-algebras. The computation of the minimal model for $\bar{A}$ is simpler than that of $A$, because we do not have to deal with the 'loop' 1.

The construction of minimal models and the above discussion extend to the case where the ground ring $k$ is no longer a field but a semi-simple ring $R$, for example a product of fields. We then consider $A_{\infty}$-algebras in the monoidal category of $R$ - $R$-bimodules.

For example, suppose that $A$ is an ordinary algebra augmented over a semi-simple ring $R$, say $A=R \oplus \bar{A}$. Then the standard complex computing $\operatorname{Ext}_{A}(R, R)$ is of the form

$$
0 \rightarrow R \stackrel{0}{\rightarrow} \operatorname{Hom}_{R}(\bar{A}, \bar{A}) \rightarrow \operatorname{Hom}_{R}\left(\bar{A} \otimes_{R} \bar{A}, \bar{A}\right) \rightarrow \ldots
$$

It has a canonical structure of dg algebra augmented over $R$. Thus the $A_{\infty}$-algebra $\operatorname{Ext}_{A}(R, R)$ may be chosen to be augmented over $R$ and, in particular, strictly unital.

Let us consider another example: Suppose that $k$ is a field and that $A$ is a unital dg $k$-algebra endowed with a decomposition

$$
1_{A}=e_{1}+\ldots+e_{N}
$$

of $1_{A}$ into idempotents $e_{i}=e_{i}^{2}$ such that the complexes $e_{j} A e_{i}$ are acyclic for $i<j$ and that $e_{i} A e_{i}$ is quasi-isomorphic to $k e_{i}$. Clearly $A$ is isomorphic to the matrix algebra

$$
\bigoplus_{i, j=1}^{N} e_{j} A e_{i}
$$

and this algebra is quasi-isomorphic to its subalgebra of upper triangular matrices

$$
B=k e_{1} \oplus \ldots \oplus k e_{N} \oplus \bigoplus_{i<j} e_{j} A e_{i} .
$$

Clearly $B$ is an augmented algebra over $R=k \times \ldots \times k$ ( $N$ factors). So we obtain 
a minimal model for $A$ as the sum of $R$ with a minimal model for

$$
\bar{B}=\bigoplus_{i<j} e_{j} A e_{i} .
$$

viewed as an $A_{\infty}$-algebra over $R$. Let $\bar{B}^{\prime}$ be this minimal model. Then we have a decomposition

$$
\bar{B}^{\prime}=\bigoplus_{i<j} H^{*}\left(e_{j} A e_{i}\right)=\bigoplus_{i<j} e_{j} H^{*}(A) e_{i}
$$

of $R$ - $R$-bimodules and the tensor power

$$
\bar{B}^{\prime} \otimes_{R} \bar{B}^{\prime} \otimes_{R} \ldots \otimes_{R} \bar{B}^{\prime} \quad(n \text { factors })
$$

is isomorphic to the sum of the

$$
e_{i_{n+1}} H^{*}(A) e_{i_{n}} \otimes_{k} e_{i_{n}} H^{*}(A) e_{i_{n-1}} \otimes \ldots \otimes_{k} e_{i_{2}} H^{*}(A) e_{i_{1}}
$$

taken over all sequences $i_{1}<i_{2}<\ldots<i_{n+1}$. These spaces vanish for $n \geqslant N$ so that in the minimal model we automatically have $m_{n}=0$ for $n \geqslant N$. For example, if $N=3$, i.e. if the unit of $A$ decomposes into $1=e_{1}+e_{2}+e_{3}$, then $\bar{B}^{\prime}$ is automatically formal. Hence $\bar{B}$ and $A$ are also formal in this case.

The following lemma presents another case where the passage to the category of augmented $A_{\infty}$-algebras is easy. A subalgebra of an $A_{\infty}$-algebra $A$ is a graded subspace $B$ such that $m_{i}$ maps $B^{\otimes i}$ to $B$ for all $i \geqslant 1$.

Lemma. Let $A$ be a strictly unital $A_{\infty}$-algebra (for example a unital dg algebra). Suppose that $H^{i} A=0$ for $i<0$ and that $H^{0} A=k$. Then there is a subalgebra $B \subset A$ whose inclusion is a quasi-isomorphism and which is augmented.

\subsection{The bar construction}

Following [46] and [16] we will restate the definitions of $A_{\infty}$-algebras and their morphisms in a more efficient way. Let $V$ be a $\mathbf{Z}$-graded vector space and let

$$
\bar{T} V=V \oplus V^{\otimes 2} \oplus \cdots
$$

be the reduced tensor algebra (in the category of graded spaces). We make $\bar{T} V$ into a graded coalgebra via the comultiplication

$$
\Delta: \bar{T} V \rightarrow \bar{T} V \otimes \bar{T} V
$$

defined by

$$
\Delta\left(v_{1}, \ldots, v_{n}\right)=\sum_{1 \leqslant i \leqslant n}\left(v_{1}, \ldots, v_{i}\right) \otimes\left(v_{i+1}, \ldots, v_{n}\right) .
$$

Note that $\Delta(v)=0$ and $\Delta\left(v_{1}, v_{2}\right)=v_{1} \otimes v_{2}$. It is then easy to see that each graded map $b: \bar{T} V \rightarrow V$ of degree 1 uniquely lifts to a graded coderivation $b: \bar{T} V \rightarrow \bar{T} V$ of degree 1 , where coderivation means that we have

$$
\Delta b=(b \otimes \mathbf{1}+\mathbf{1} \otimes b) \Delta .
$$

To see that this is plausible, one should remember that algebra derivations $\bar{T} V \rightarrow$ $\bar{T} V$ are in bijection with maps $V \rightarrow \bar{T} V$. The component of $b$ that maps $V^{\otimes n}$ to 
$V^{\otimes u}$ is

$$
\mathbf{1}^{\otimes r} \otimes b_{s} \otimes \mathbf{1}^{\otimes t}
$$

where $r+1+t=u$. Thus we have a bijection between coderivations $b: \bar{T} V \rightarrow \bar{T} V$ of degree 1 and families of maps $b_{n}: V^{\otimes n} \rightarrow V, n \geqslant 1$, of degree 1 .

Now let $A$ be another $\mathbf{Z}$-graded space and let $S A$ be its suspension: $(S A)^{p}=$ $A^{p+1}$. Define a bijection between the families of maps $b_{n}:(S A)^{\otimes n} \rightarrow S A$ of degree 1 and maps $m_{n}: A^{\otimes n} \rightarrow A, n \geqslant 1$, by the commutative square

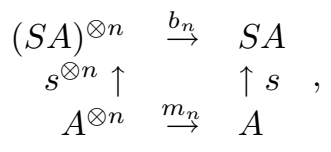

where $s: A \rightarrow S A$ is the canonical map $a \mapsto a$ of degree -1 . Note that $m_{n}$ is of degree $-n+1-(-1)=2-n$. Then we have the following easy

Lemma. The following are equivalent

(i) The maps $m_{n}: A^{\otimes n} \rightarrow$ A yield an $A_{\infty}$-algebra structure on $A$.

(ii) The coderivation $b: \bar{T} S A \rightarrow \bar{T} S A$ satisfies $b^{2}=0$.

(iii) For each $n \geqslant 1$, we have

$$
\sum b_{u}\left(\mathbf{1}^{\otimes r} \otimes b_{s} \otimes \mathbf{1}^{\otimes t}\right)=0
$$

where the sum runs over all decompositions $n=r+s+t$ and we put $u=r+1+t$.

Note that no signs appear in the last formula. The lemma yields a bijection between $A_{\infty}$-algebra structures on $A$ and coalgebra differentials on $\bar{T} S A$. It is also easy to check that if $A$ and $B$ are $A_{\infty}$-algebras, then morphisms of $A_{\infty}$-algebras $f: A \rightarrow B$ are in bijection with morphisms of dg coalgebras $\bar{T} S A \rightarrow \bar{T} S B$.

\subsection{Homotopy equivalences and quasi-isomorphisms}

Let $A$ and $B$ be $A_{\infty}$-algebras and $f, g$ morphisms from $A$ to $B$. Let $F, G$ denote the corresponding morphisms of $\mathrm{dg}$ coalgebras $\bar{T} S A \rightarrow \bar{T} S B$. One defines $f$ and $g$ to be homotopic if there is a homogeneous map $H: \bar{T} S A \rightarrow \bar{T} S B$ of degree -1 such that

$$
\Delta H=F \otimes H+H \otimes G \text { and } \quad F-G=b \circ H+H \circ b .
$$

It is easy to translate this into the existence of a family of maps $h_{n}: A^{\otimes n} \rightarrow B$ satisfying suitable conditions.

Theorem (Prouté [38], cf. also [18]). (a) Homotopy is an equivalence relation on the set of morphisms of $A_{\infty}$-algebras $A \rightarrow B$.

(b) A morphism of $A_{\infty}$-algebras $s: A \rightarrow B$ is a quasi-isomorphism iff it is an homotopy equivalence (i.e. it becomes an isomorphism in the category obtained by dividing the category of $A_{\infty}$-algebras by the homotopy relation). 


\section{4. $A_{\infty}$-modules and the derived category}

\subsection{Reminder on the derived category}

We refer to $[\mathbf{2 0}, \mathrm{Ch}$. I] for a concise presentation of derived categories and to [51] and $[\mathbf{1 0}]$ for more leisurely introductions. We refer to [44] for foundational material on resolutions of unbounded complexes.

Let $A$ be an associative $k$-algebra with 1 and denote by $\operatorname{Mod} A$ the category of unital right $A$-modules. The derived category $\mathcal{D} A$ is classically constructed $[\mathbf{4 9}][\mathbf{5 0}]$ in three steps:

1st step: We define the category $\mathcal{C} A$ to be the category of complexes

$$
\ldots \rightarrow M^{p} \rightarrow M^{p+1} \rightarrow \ldots
$$

with morphisms of complexes as morphisms.

2nd step: We define the homotopy category $\mathcal{H} A$. Its objects are the same as those of $\mathcal{C} A$. The group of morphisms $M \rightarrow N$ in $\mathcal{H} A$ is the group of morphisms of complexes divided by the subgroup of null homotopic morphisms.

3rd step: We define the derived category $\mathcal{D} A$ to be the localization of the homotopy category $\mathcal{H} A$ with respect to all quasi-isomorphisms. Thus the objects of $\mathcal{D} A$ are the same as those of $\mathcal{H} A$ and its morphisms are obtained from morphisms in the homotopy category by formally inverting all quasi-isomorphisms.

To get an idea of what the morphisms in the derived category are it is helpful to consider the following special case: Let $M$ and $N$ be two $A$-modules. Identify $M$ with the complex

$$
\ldots \rightarrow 0 \rightarrow M \rightarrow 0 \rightarrow \ldots
$$

concentrated in degree 0 . For any complex $K$ and any $n \in \mathbf{Z}$, denote by $K[n]$ the complex $K$ shifted by $n$ degrees to the left: $K[n]^{p}=K^{n+p}$ and $d_{K[n]}=(-1)^{n} d_{K}$. Then there is a canonical quasi-isomorphism

$$
\operatorname{Hom}_{\mathcal{D} A}(M, N[n])=\operatorname{Ext}_{A}^{n}(M, N)
$$

valid for any $n \in \mathbf{Z}$ if we take the right hand side to vanish for negative $n$.

\subsection{Generalization to $A_{\infty}$-algebras}

Let $A$ be an $A_{\infty}$-algebra. We generalize the above step by step.

1st step: An $A_{\infty}$-module over $A$ (cf. [17]) is a $\mathbf{Z}$-graded space $M$ endowed with maps

$$
m_{n}^{M}: M \otimes A^{\otimes n-1} \rightarrow M, n \geqslant 1,
$$

of degree $2-n$ such that an identity of the same form as the one in the definition (3.1) of an $A_{\infty}$-algebra holds. However, the term

$$
m_{u}\left(\mathbf{1}^{\otimes r} \otimes m_{s} \otimes \mathbf{1}^{\otimes t}\right)
$$


has to be interpreted as

$$
m_{u}^{M}\left(\mathbf{1}^{\otimes r} \otimes m_{s} \otimes \mathbf{1}^{\otimes t}\right)
$$

if $r>0$ and as

$$
m_{u}^{M}\left(m_{s}^{M} \otimes \mathbf{1}^{\otimes t}\right)
$$

if $r=0$. Equivalently, the datum of an $A_{\infty}$-structure on a graded space $M$ is the datum of a differential $m_{1}^{M}$ on $M$ and of a morphism of $A_{\infty}$-algebras from $A$ to the opposite of the dg algebra $\operatorname{Hom}_{k}{ }_{k}(M, M)$ (cf. 3.3). A morphism of $A_{\infty}$-modules $f: L \rightarrow M$ is a sequence of graded morphisms

$$
f_{n}: L \otimes A^{\otimes n-1} \rightarrow M
$$

of degree $1-n$ such that for each $n \geqslant 1$, we have

$$
\sum(-1)^{r+s t} f_{u} \circ\left(\mathbf{1}^{\otimes r} \otimes m_{s} \otimes \mathbf{1}^{\otimes t}\right)=\sum(-1)^{(r+1) s} m_{u} \circ\left(f_{r} \otimes \mathbf{1}^{\otimes s}\right),
$$

where the left hand sum is taken over all decompositions $n=r+s+t, r, t \geqslant 0, s \geqslant 1$ and we put $u=r+1+t$; and the right hand sum is taken over all decompositions $n=r+s, r \geqslant 1, s \geqslant 0$ and we put $u=1+s$. The morphism $f$ is a quasiisomorphism if $f_{1}$ is a quasi-isomorphism. The identical morphism $f: M \rightarrow M$ is given by $f_{1}=\mathbf{1}_{M}, f_{i}=0$, for all $i>0$. The composition $f g$ is given by

$$
(f g)_{n}=\sum(-1)^{(r-1) s} f_{u}\left(g_{r} \otimes \mathbf{1}^{\otimes s}\right)
$$

where the sum runs over all decompositions $n=r+s$ and we put $u=1+s$.

In analogy with (3.6), one can easily check that $A_{\infty}$-structures on a graded space $M$ are in bijection with comodule differentials on $S M \otimes T S A$, where $T S A$ is the coaugmented tensor coalgebra on $S A$ :

$$
T S A=k \oplus \bar{T} S A .
$$

The comultiplication on $T S A$ is given by

$$
\Delta(x)=1 \otimes x+\Delta_{\bar{T} S A}(x)+x \otimes 1 .
$$

The differential of $T S A$ is induced by $b$ on $\bar{T} S A$ and maps $k$ to zero. The comodule structure on $S M \otimes T S A$ is induced from the coalgebra structure on $T S A$. Morphisms of $A_{\infty}$-modules then correspond bijectively to morphisms of dg comodules.

We define $\mathcal{C}_{\infty} A$ to be the category of $A_{\infty}$-modules. If $A$ is an ordinary associative algebra, we have a faithful functor

$$
\mathcal{C} A \rightarrow \mathcal{C}_{\infty} A
$$

but $\mathcal{C}_{\infty} A$ has more objects and more morphisms than $\mathcal{C} A$. A typical example of an $A_{\infty}$-module over $A$ is obtained as follows: Suppose that $A$ is the endomorphism algebra of a module $M^{\prime}$ over an ordinary algebra $B$ and let $M$ be a projective resolution of $M^{\prime}$ over $B$. Then it is well-known that each element of $A$ lifts to an endomorphism of the complex $M$ and this endomorphism is unique up to homotopy. The resulting 'action up to homotopy' of $A$ on $M$ may be shown to arise from a structure of $A_{\infty}$-module, cf. [22,6.1]. 
2nd step: By definition, a morphism $f: L \rightarrow M$ of $A_{\infty}$-modules is nullhomotopic if there is a family of graded maps

$$
h_{n}: L \otimes A^{\otimes n-1} \rightarrow M, n \geqslant 1,
$$

homogeneous of degree $-n$ such that

$$
\begin{aligned}
f_{n}= & \sum(-1)^{r s} m_{1+s} \circ\left(h_{r} \otimes \mathbf{1}^{\otimes s}\right) \\
& +\sum(-1)^{r+s t} h_{u} \circ\left(\mathbf{1}^{\otimes r} \otimes m_{s} \otimes \mathbf{1}^{\otimes t}\right),
\end{aligned}
$$

where the first sum runs over all decompositions $n=r+s, r \geqslant 1, s \geqslant 0$ and the second sum over all decompositions $n=r+s+t, r, t \geqslant 0, s \geqslant 1$ and we put $u=r+1+t$.

The homotopy category $\mathcal{H}_{\infty} A$ has the same objects as $\mathcal{C}_{\infty} A$ and morphisms from $L$ to $M$ are morphisms of $A_{\infty}$-modules modulo nullhomotopic morphisms.

3rd step: To define the derived category, we should formally invert all quasiisomorphisms. This turns out not to be necessary:

Theorem. Each quasi-isomorphism of $A_{\infty}$-modules is an homotopy equivalence.

We therefore define the derived category $\mathcal{D}_{\infty} A$ to be equal to the homotopy category $\mathcal{H}_{\infty} A$. If $k$ is only assumed to be a commutative ring, the assertion of the theorem is no longer true in general and the derived category is different from the homotopy category.

\subsection{Solution of problem 1}

Theorem. Let $A$ be an $A_{\infty}$-algebra.

a) If $A$ is an ordinary associative algebra with 1 , the canonical functor

$$
\mathcal{D} A \rightarrow \mathcal{D}_{\infty} A
$$

is an equivalence onto the full subcategory of homologically unital $A_{\infty}$-modules, i.e. $A_{\infty}$-modules $M$ such that $1 \in A$ acts by the identity on $H^{*} M$.

b) For each $A_{\infty}$-module $M$, the graded space $H^{*} M$ admits an $A_{\infty}$-module structure such that $m_{1}=0$ and $M$ is isomorphic to $H^{*} M$ in $\mathcal{D}_{\infty} A$. It is unique up to (non unique, non strict) isomorphism of $A_{\infty}$-modules.

The $A_{\infty}$-module $H^{*} M$ of b) is called the minimal model of $M$. The theorem explains the answer to problem 1: Indeed, if $M$ is a complex over an algebra $A$ as in (2.1), then we may view it as an $A_{\infty}$-module, or, in other words, consider its image in $\mathcal{D}_{\infty} A$. By b), the image is isomorphic to $H^{*} M$ endowed with its canonical $A_{\infty^{-}}$ structure $m_{1}=0, m_{2}, m_{3}, \ldots$. The full faithfulness of the functor $\mathcal{D} A \rightarrow \mathcal{D}_{\infty} A$ then shows that $\left(H^{*} M, m_{1}=0, m_{2}, \ldots\right)$ determines the complex $M$ up to isomorphism in the derived category $\mathcal{D} A$.

In fact the theorem tells us more: If we also use the essential surjectivity of the functor $\mathcal{D} A \rightarrow \mathcal{D}_{\infty} A$ onto its image, we see that if $V$ is a graded vector space, then we have a bijection between complexes of $A$-modules $M$ with $H^{*} M \stackrel{\sim}{\rightarrow} V$ 
(as graded vector spaces) up to isomorphism in $\mathcal{D} A$, and homologically unital $A_{\infty^{-}}$ structures $m_{1}=0, m_{2}, m_{3}, m_{4}, \ldots$ on $V$ up to isomorphism of such structures in $\mathcal{C}_{\infty} \mathcal{A}$. Note that the latter set is naturally the set of orbits under the action of a group, namely the group of families $f_{i}, i \geqslant 1$, as in (4.1) acting on the set of homologically unital module structures by a conjugation action deduced from (4.2). If the total dimensions of $V$ and $A$ are finite, we even obtain an algebraic group acting on an algebraic variety.

\section{Triangulated structure}

\subsection{Towards problem 2}

Consider problem 2 of (2.1) for the special case $n=1$ : We then have an ordinary associative algebra $B$ with 1 and a $B$-module $M$ and we wish to reconstruct the category filt $M \subset \operatorname{Mod} B$ of iterated extensions of $M$ by itself from the extension algebra

$$
E=\operatorname{Ext}_{B}^{*}(M, M)
$$

with its canonical $A_{\infty}$-structure. The basic idea is to realize filt $M$ as a subcategory of the derived category of $E$. In fact, this subcategory will simply be the subcategory of iterated extensions of the free $E$-module of rank one by itself. Now the derived category is not abelian in general so that a priori it makes no sense to speak of extensions. However, like the derived category of an ordinary associative algebra, the derived category of an $A_{\infty}$-algebra does admit a triangulated structure and this suffices for our purposes.

\subsection{Triangulated structure on $\mathcal{D}_{\infty} A$}

Recall that a triangulated category is an additive category $\mathcal{T}$ endowed with an additive endofunctor $S: \mathcal{T} \rightarrow \mathcal{T}$ called its suspension (or shift) and with a class of sequences of the form

$$
X \rightarrow Y \rightarrow Z \rightarrow S X \quad(*)
$$

called triangles (or distinguished triangles). These have to satisfy a number of axioms (see the references at the beginning of 4.1). If $Y$ occurs in a triangle $(*)$, it is called an extension of $Z$ by $X$.

For example, if $A$ is an ordinary associative algebra with 1 , the derived category $\mathcal{D} A$ is triangulated: The suspension of a complex $K$ is defined by

$$
(S K)^{p}=K^{p+1}, d_{S K}=-d_{K} .
$$

Each short exact sequence of complexes

$$
0 \rightarrow K \rightarrow L \rightarrow M \rightarrow 0
$$

gives rise to a canonical triangle

$$
K \rightarrow L \rightarrow M \rightarrow S K
$$

and (up to isomorphism) all triangles are obtained in this way. This generalizes to $A_{\infty}$-algebras as follows 
Proposition. Let $A$ be an $A_{\infty}$-algebra. Then the derived category $\mathcal{D}_{\infty} A$ admits a triangulated structure with suspension functor $S$ defined by

$$
(S M)^{p}=M^{p+1}, m_{n}^{S M}=(-1)^{n} m_{n}^{M}
$$

and such that each short exact sequence

$$
0 \rightarrow K \stackrel{i}{\rightarrow} L \stackrel{p}{\rightarrow} M \rightarrow 0
$$

of $A_{\infty}$-modules with strict morphisms $i$ and $p$ gives rise to a canonical triangle. Up to isomorphism in $\mathcal{D}_{\infty} A$, all triangles are obtained in this way.

\section{Standard functors}

\subsection{More on problem 2}

If $\mathcal{T}$ is a triangulated category and $M$ an object of $\mathcal{T}$, we denote by filt $M=$ filt $\mathcal{T} M$ the full subcategory of $\mathcal{T}$ whose objects are iterated extensions of $M$ by itself. We denote by tria $M$ the closure of $M$ under suspension, desuspension and forming extensions. Thus tria $M$ is the smallest triangulated subcategory of $\mathcal{T}$ containing $M$ and filt $M$ is a full subcategory of tria $M$.

If $\mathcal{S}$ and $\mathcal{T}$ are triangulated categories a triangle functor $\mathcal{S} \rightarrow \mathcal{T}$ is an additive functor $F: \mathcal{S} \rightarrow \mathcal{T}$ together with an isomorphism of functors $\varphi: F S \rightarrow S F$ such that for each triangle $(X, u, Y, v, Z w)$ of $\mathcal{S}$, the sequence

$$
F X \stackrel{F u}{\longrightarrow} F Y \stackrel{F v}{\longrightarrow} F Z \stackrel{(\varphi X)(F w)}{\longrightarrow} S F X
$$

is a triangle of $\mathcal{T}$. If $M$ is an object of $\mathcal{T}$ and $F$ a triangle functor then clearly $F$ tria $M \subset$ tria $F M$ and similarly for filt $M$.

In the situation of (5.1), consider the subcategory filt $E \subset \mathcal{D}_{\infty} E$ obtained by taking the closure under extensions of the free $E$-module of rank one (also denoted by $E$ ). We would like to show that the subcategory filt $E$ is equivalent to filt $\operatorname{Mod}_{B} M$. For this, we first observe that the inclusion

$$
\operatorname{Mod} B \rightarrow \mathcal{D} B
$$

induces an equivalence

$$
\text { filt }_{\operatorname{Mod} B} M \stackrel{\sim}{\rightarrow} \text { filt }_{\mathcal{D} B} M .
$$

It will thus suffice to construct a triangle functor $\mathcal{D} B \rightarrow \mathcal{D}_{\infty} E$ inducing an equivalence from filt $M$ onto filt $E$. The following diagram summarizes the setup:

$$
\begin{array}{ccccc}
\operatorname{Mod} B & \rightarrow & \mathcal{D} B & \rightarrow & \mathcal{D}_{\infty} E \\
\uparrow & & \uparrow & & \uparrow \\
\text { filt } M & \stackrel{\sim}{\rightarrow} & \text { filt }_{\mathcal{D} B} M & \stackrel{\sim}{\rightarrow} & \text { filt } E
\end{array}
$$

The triangle functor $\mathcal{D} B \rightarrow \mathcal{D}_{\infty} E$ is constructed as a composition of standard functors. 


\subsection{Restriction}

Let $f: A \rightarrow B$ be a morphism of $A_{\infty}$-algebras and let $M$ be a module over $B$. Define

$$
m_{n}^{A}: M \otimes A^{\otimes n-1} \rightarrow M, n \geqslant 1
$$

by

$$
m_{n}^{A}=\sum(-1)^{s} m_{r+1}\left(\mathbf{1} \otimes f_{i_{1}} \otimes \cdots \otimes f_{i_{r}}\right)
$$

where the sum runs over all $1 \leqslant r \leqslant n-1$ and all decompositions $n-1=i_{1}+\cdots+i_{r}$ and the sign is like in (3.4). Then it is not hard to check that the $m_{n}^{A}$ define an $A_{\infty}$-module $f^{*} M$ over $A$. The assignment $M \rightarrow f^{*} M$ becomes a functor

$$
\mathcal{C}_{\infty} B \rightarrow \mathcal{C}_{\infty} A
$$

and induces a triangle functor

$$
\mathcal{D}_{\infty} B \rightarrow \mathcal{D}_{\infty} A
$$

which will still be denoted by $f^{*}$.

Proposition. The functor $f^{*}: \mathcal{D}_{\infty} A \rightarrow \mathcal{D}_{\infty} B$ is an equivalence if $f$ is a quasiisomorphism.

\subsection{Hom and Tensor}

Let $B$ be an $A_{\infty}$-algebra and $X$ and $M$ two $B$-modules. For $n \in \mathbf{Z}$, let $\mathrm{Hom}_{B}^{\infty}(X, M)$ be the space of graded comodule maps $f: S X \otimes T S B \rightarrow S M \otimes T S B$ of degree $n$ and define

$$
d(f)=b \circ f-(-1)^{n} f \circ b .
$$

Then $\operatorname{Hom}_{B}^{\infty}(X, M)$ is a complex which is bifunctorial in $X$ and $M$. If $B$ is an ordinary algebra and $X, M$ are ordinary modules, then $\mathrm{H}_{\circ}^{\infty} \mathbf{m}_{B}^{\bullet}(X, M)$ computes $\operatorname{Ext}_{B}^{*}(X, M)$.

Now suppose that $A$ is another $A_{\infty}$-algebra and that the $B$-module structure on $X$ comes from an $A$ - $B$-bimodule structure, i.e. $X$ is endowed with graded maps

$$
m_{t, u}: A^{\otimes t-1} \otimes X \otimes B^{\otimes u-1} \rightarrow X, t, u \geqslant 1,
$$

of degree $3-t-u$ such that we have the associativity identity of (3.1), where the terms $\mathbf{1}^{\otimes r} \otimes m_{s} \otimes \mathbf{1}^{\otimes t}$ have to be interpreted according to the type of their arguments. Then $\mathrm{Hom}_{B}^{\infty}(X, M)$ becomes a (right) $A_{\infty}$-module over $A$ and we have the Hom-functor

$$
\mathrm{Hom}_{B}^{\infty}(X, ?): \mathcal{C}_{\infty} B \rightarrow \mathcal{C}_{\infty} A
$$

Proposition. The Hom-functor admits a left adjoint

$$
? \stackrel{\infty}{\otimes}_{A} X: \mathcal{C}_{\infty} A \rightarrow \mathcal{C}_{\infty} B
$$

We have an induced pair of adjoint triangle functors

$$
\begin{aligned}
& \mathcal{D}_{\infty} A \\
& ? \stackrel{\infty}{\otimes_{A}} X \underset{\mathcal{D}_{\infty} B}{\stackrel{\downarrow}{\downarrow \uparrow}} \operatorname{Hom}_{B}^{\infty}(X, ?)
\end{aligned}
$$


It is not hard to make $L \stackrel{\infty}{\otimes}_{A} X$ explicit. Its underlying graded space is $L \otimes_{k}$ $T S A \otimes_{k} X$. If $A$ is an ordinary associative algebra with 1 and $L, X$ are ordinary modules, then $L \stackrel{\infty}{\otimes}_{A} X$ is a complex computing $\operatorname{Tor}_{*}^{A}(L, X)$.

\subsection{Application to problem 2}

Let $B$ be an $A_{\infty}$-algebra and $M$ a $B$-module. Then

$$
A=\operatorname{Hom}_{B}^{\infty}(M, M)
$$

is a dg algebra and in particular an $A_{\infty}$-algebra. The module $M$ becomes an $A$ - $B$ bimodule.

Proposition. The functor

$$
F=\mathrm{Hom}_{B}^{\infty}(M, ?): \mathcal{D}_{\infty} B \rightarrow \mathcal{D}_{\infty} A
$$

induces a triangle equivalence

$$
\text { tria } M \stackrel{\sim}{\rightarrow} \text { tria } A
$$

taking $M$ to $A$ and thus an equivalence filt $M \stackrel{\sim}{\rightarrow}$ filt $A$.

Now consider the situation of (5.1). In this case, $A$ is a dg algebra whose homology is the extension algebra of $M$. Let us simply write

$$
E=\operatorname{Ext}_{B}^{*}(M, M)
$$

for the extension algebra endowed with its canonical structure of $A_{\infty}$-algebra. Then we know by Theorem 3.3 that we have a quasi-isomorphism $E \rightarrow A$. This yields the restriction functor

$$
\text { res : } \mathcal{D}_{\infty} A \rightarrow \mathcal{D}_{\infty} E
$$

which is an equivalence by Proposition 6.2. Consider the square

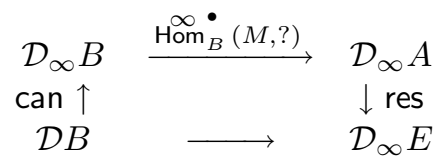

The left vertical arrow induces an equivalence onto its image and maps $M$ to $M$. By the above proposition, the top horizontal arrow induces an equivalence filt $M \stackrel{\sim}{\rightarrow}$ filt $A$. The right vertical arrow is an equivalence mapping the free module $A$ to the free module $E$ and hence induces an equivalence filt $A \stackrel{\sim}{\rightarrow}$ filt $E$. It follows that the bottom arrow induces an equivalence filt $\mathcal{D}_{B} M \stackrel{\sim}{\rightarrow}$ filt $E$. Finally, we know that filt ${ }_{\mathcal{D} B} M$ is equivalent to filt $M \subset \operatorname{Mod} B$ so that we do obtain the required equivalence between filt $E \subset \mathcal{D}_{\infty} E$ and filt $M \subset \operatorname{Mod} B$.

\subsection{Solution of the original problem 2}

In order to treat problem 2 as it is stated in (2.1) with $n$ modules $M_{1}, \ldots, M_{n}$, let $e_{i}$ be the idempotent of $E$ corresponding to the direct factor $M_{i}$ of $M=\bigoplus M_{i}$. There is a canonical isomorphism

$$
E^{0} \stackrel{\sim}{\rightarrow} \operatorname{Hom}_{\mathcal{D}_{\infty} E}(E, E)
$$


and thus $e_{i}$ yields an idempotent endomorphism of $E$ in the derived category. Now the derived category is a triangulated category with infinite sums so that by a wellknown trick [4], the idempotent $e_{i}$ splits in $\mathcal{D}_{\infty} E$. Let $X_{i}$ denote its image. We have

$$
E \stackrel{\sim}{\rightarrow} \bigoplus X_{i}
$$

in $\mathcal{D}_{\infty} E$. The functor constructed in 6.4 sends $X_{i}$ to $M_{i}$ and thus induces an equivalence filt $X_{i} \stackrel{\sim}{\rightarrow}$ filt $M_{i}$.

\section{Twisted objects}

\subsection{Motivation}

Let $A$ be an associative algebra with 1 and tria $A$ the triangulated subcategory of $\mathcal{D} A$ generated by the free $A$-module of rank one (as in 6.1). Up to isomorphism, the objects of tria $A$ are the bounded complexes of finitely generated free $A$-modules and the morphisms between two such complexes are in bijection with the homotopy classes of morphisms of complexes. In other words, tria $A$ is triangle equivalent to the bounded homotopy category of finitely generated free $A$-modules. The advantage of this description of tria $A$ is that morphisms and objects are readily computable. The aim of this section is to generalize this description to any $A_{\infty}$-algebra $A$ with a strict unit (cf. below). The generalization will be of the form

$$
\text { tria } A \stackrel{\sim}{\rightarrow} H^{0} \text { tw } A
$$

where tw $A$ is the $A_{\infty}$-category of twisted objects over $A$. An $A_{\infty}$-category is an ' $A_{\infty}$-algebra with several objects' analogous to a 'ring with several objects' [33]. The above equivalence illustrates the general fact that working with several objects is natural and useful. From the outset, we will therefore replace the $A_{\infty}$-algebra $A$ by an $A_{\infty}$-category with strict identities $\mathcal{A}$. We will then have more generally

$$
\operatorname{tria} \mathcal{A} \stackrel{\sim}{\rightarrow} H^{0} \operatorname{tw} \mathcal{A} \text {. }
$$

The material in this section is adapted from [23], [25]. The special case of $\mathrm{dg}$ categories goes back to Bondal-Kapranov's article [5].

\section{2. $A_{\infty}$-categories}

Let $k$ be a field. An $A_{\infty}$-category $\mathcal{A}$ is the datum of

- a class of objects obj $(\mathcal{A})$,

- for all $A, B \in \mathcal{A}$ (we write $A \in \mathcal{A}$ instead of $A \in \operatorname{obj}(\mathcal{A})$ ), a $\mathbf{Z}$-graded vector space $\operatorname{Hom}_{\mathcal{A}}(A, B)$, often denoted by $(A, B)$,

- for all $n \geqslant 1$ and all $A_{0}, \ldots, A_{n} \in \mathcal{A}$, a graded map

$$
m_{n}:\left(A_{n-1}, A_{n}\right) \otimes\left(A_{n-2}, A_{n-1}\right) \otimes \cdots \otimes\left(A_{0}, A_{1}\right) \rightarrow\left(A_{0}, A_{n}\right)
$$

of degree $2-n$

such that for each $n \geqslant 1$ and all $A_{0}, \ldots, A_{n} \in \mathcal{A}$, we have the identity

$$
\sum(-1)^{r+s t} m_{u}\left(\mathbf{1}^{\otimes r} \otimes m_{s} \otimes \mathbf{1}^{\otimes t}\right)=0
$$


of maps

$$
\left(A_{n-1}, A_{n}\right) \otimes\left(A_{n-2}, A_{n-1}\right) \otimes \cdots \otimes\left(A_{0}, A_{1}\right) \rightarrow\left(A_{0}, A_{n}\right),
$$

where the sum runs over all decompositions $n=r+s+t$ and we put $u=r+1+t$.

For example, the datum of an $A_{\infty}$-category $\mathcal{A}$ with one object $*$ is equivalent to the datum of the endomorphism $A_{\infty}$-algebra $\operatorname{Hom}_{\mathcal{A}}(*, *)$. Another class of examples is provided by dg categories : these are exactly the $A_{\infty}$-categories whose composition maps $m_{n}$ vanish for all $n \geqslant 3$.

Note that for two reasons, an $A_{\infty}$-category is not a category in general : firstly, the composition $m_{2}$ may not be associative; secondly, there may not be identity morphisms.

Let $\mathcal{A}$ be an $A_{\infty}$-category and $A \in \mathcal{A}$. A morphism $e_{A} \in \operatorname{Hom}_{\mathcal{A}}^{0}(A, A)$ is a strict identity if we have

$$
m_{2}\left(f, e_{A}\right)=f, m_{2}\left(e_{A}, g\right)=g
$$

whenever these make sense and

$$
m_{n}\left(\ldots, e_{A}, \ldots\right)=0
$$

for all $n \neq 2$. In particular, $e_{A}$ is then a cycle of the complex $\left(\operatorname{Hom}_{\mathcal{A}}(A, A), m_{1}\right)$. Clearly, if $e_{A}$ exists, it is unique. The $A_{\infty}$-category $\mathcal{A}$ has strict identities if there is a strict identity $e_{A}$ for each object $A \in \mathcal{A}$.

\section{3. $A_{\infty}$-functors}

Let $\mathcal{A}, \mathcal{B}$ be two $A_{\infty}$-categories. An $A_{\infty}$-functor $F: \mathcal{A} \rightarrow \mathcal{B}$ is the datum of

- a map $F: \operatorname{obj}(\mathcal{A}) \rightarrow \operatorname{obj}(\mathcal{B})$,

- for all $n \geqslant 1$ and all $A_{0}, \ldots, A_{n} \in \mathcal{A}$ a graded map

$$
F_{n}:\left(A_{n-1}, A_{n}\right) \otimes\left(A_{n-2}, A_{n-1}\right) \otimes \cdots \otimes\left(A_{0}, A_{1}\right) \rightarrow \operatorname{Hom}_{\mathcal{B}}\left(F A_{0}, F A_{n}\right)
$$

of degree $1-n$

such that conditions analogous to those of (3.4) hold. The $A_{\infty}$-functor $F$ is strict if we have $F_{n}=0$ for all $n \geqslant 2$. This implies that $F_{1}$ 'commutes with all compositions'.

\section{4. $\quad$ Modules}

We leave it as an exercise to the reader to generalize (4.2) to $A_{\infty}$-categories. In particular, if $\mathcal{A}$ is an $A_{\infty}$-category, he may

- define the category $\mathcal{C}_{\infty} \mathcal{A}$ of $\mathcal{A}$-modules

- define the derived category $\mathcal{D}_{\infty} \mathcal{A}$

- define a structure of dg category

$$
\mathrm{H}^{\infty} \mathrm{m}(L, M), L, M \in \mathcal{C}_{\infty} \mathcal{A}
$$

on $\mathcal{C}_{\infty} \mathcal{A}$ as in 6.3

- define the Yoneda functor

$$
Y: \mathcal{A} \rightarrow \mathcal{C}_{\infty} \mathcal{A}, A \mapsto \mathcal{A}(?, A) .
$$


Here we view $\mathcal{C}_{\infty} \mathcal{A}$ as a dg category (and hence an $A_{\infty}$-category) and the Yoneda functor is to be an $A_{\infty}$-functor.

\subsection{Factorization of the Yoneda functor}

Let $\mathcal{A}$ be an $A_{\infty}$-category and let tria $\mathcal{A}$ denote the triangulated subcategory of $\mathcal{D}_{\infty} \mathcal{A}$ generated by the image of the Yoneda functor.

Theorem. Suppose that $\mathcal{A}$ has strict identities. Then there is an $A_{\infty}$-category tw $\mathcal{A}$ and a factorization

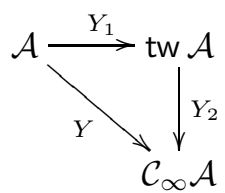

of the Yoneda functor $Y$ such that $Y_{1}$ is a strict fully faithful $A_{\infty}$-functor and $Y_{2}$ is an $A_{\infty}$-functor inducing an equivalence

$$
H^{0} \text { tw } \mathcal{A} \stackrel{\sim}{\rightarrow} \operatorname{tria} \mathcal{A} \subset \mathcal{D}_{\infty} \mathcal{A} .
$$

The theorem may be enhanced so as to characterize the category tw $\mathcal{A}$ of twisted objects by a universal property.

\subsection{Explicit description of tw $\mathcal{A}$}

Let $\mathcal{A}$ be an $A_{\infty}$-category with strict identities. We will explicitly construct the category of twisted objects tw $\mathcal{A}$ in two steps. First, we construct the $A_{\infty}$-category $\mathbf{Z} \mathcal{A}$, which is the 'closure under shifts' of $\mathcal{A}$ : the objects of $\mathbf{Z} \mathcal{A}$ are the pairs $(A, n)$ of an object $A \in \mathcal{A}$ and an integer $n \in \mathbf{Z}$. The morphisms from $(A, n)$ to $\left(A^{\prime}, n^{\prime}\right)$ are in bijection with the elements of the graded space

$$
\operatorname{Hom}_{\mathcal{A}}\left(A, A^{\prime}\right)\left[n^{\prime}-n\right] .
$$

We will identify $\mathcal{A}$ with the full sub- $A_{\infty}$-category of $\mathbf{Z} \mathcal{A}$ on the objects $(A, 0)$. The compositions of $\mathbf{Z} \mathcal{A}$ are obtained by shifting the compositions of $\mathcal{A}$. The reader is invited to write down an explicit formula with correct signs.

In a second step, we construct tw $\mathcal{A}$ as the 'closure under extensions' of $\mathbf{Z} \mathcal{A}$ : Objects of tw $\mathcal{A}$ are pairs $(B, \delta)$, where $B$ is a sequence $\left(A_{1}, \ldots, A_{r}\right)$ of objects of $\mathbf{Z} \mathcal{A}$, and $\delta=\left(\delta_{i j}\right)$ a matrix of morphisms of degree 1

$$
\delta_{i j} \in \operatorname{Hom}_{\mathbf{Z} \mathcal{A}}^{1}\left(A_{j}, A_{i}\right)
$$

such that we have $\delta_{i j}=0$ for all $i \geqslant j$ and

$$
\sum_{t=1}^{\infty}(-1)^{\frac{t(t-1)}{2}} m_{t}(\delta, \delta, \ldots, \delta)=0 .
$$

Here we denote by $m_{n}$ the extension of $m_{n}^{\mathbf{Z} \mathcal{A}}$ to matrices. Note that since $\delta$ is strictly upper triangular, the sum contains only finitely many non zero terms. The strange sign in the formula disappears in the $b_{n}$-language (3.6). 
Alternatively, we can view an object $(B, \delta)$ as the datum of an object $B$ of the additive hull of $\mathbf{Z} \mathcal{A}$ endowed with a finite split filtration and with an endomorphism $\delta$ of degree 1 which strictly decreases the filtration.

The space of morphisms from $(B, \delta)$ to $\left(B^{\prime}, \delta^{\prime}\right)$ is defined as

$$
\bigoplus_{i, j} \operatorname{Hom}_{\mathbf{Z} \mathcal{A}}\left(A_{i}, A_{j}^{\prime}\right) \text {. }
$$

Let $n \geqslant 1$ and let $\left(B_{0}, \delta_{0}\right), \ldots,\left(B_{n}, \delta_{n}\right)$ be objects of tw $\mathcal{A}$. We will define the composition map (where the $\delta_{i}$ are suppressed from the notation)

$$
m_{n}:\left(B_{n-1}, B_{n}\right) \otimes \cdots \otimes\left(B_{0}, B_{1}\right) \rightarrow\left(B_{0}, B_{n}\right) .
$$

In the following formula, we write $\delta$ for all $\delta_{i}$

$$
m_{n}^{\mathrm{tw} \mathcal{A}}=\sum_{t=0}^{\infty} \sum \pm m_{n+t}^{\mathbf{Z} \mathcal{A}} \circ\left(\mathbf{1}^{\otimes i_{1}} \otimes \delta^{\otimes j_{1}} \otimes \mathbf{1}^{\otimes i_{2}} \otimes \delta^{\otimes j_{2}} \otimes \ldots \otimes \mathbf{1}^{\otimes i_{r}} \otimes \delta^{\otimes j_{r}}\right) .
$$

Here the terms of the second sum are in bijection with the non-commutative monomials

$$
X^{i_{1}} Y^{j_{1}} \ldots X^{i_{r}} Y^{j_{r}}
$$

of degree $n$ in $X$ and degree $t$ in $Y$ and the sign is given by the identity

$$
(S X)^{i_{1}}(S Y)^{j_{1}} \ldots(S X)^{i_{r}}(S Y)^{j_{r}}= \pm S^{n+t} X^{i_{1}} Y^{j_{1}} \ldots X^{i_{r}} Y^{j_{r}}
$$

in the algebra $k<X, Y, S>/(S X-X S, S Y+Y S)$. The pictorial version of the formula is

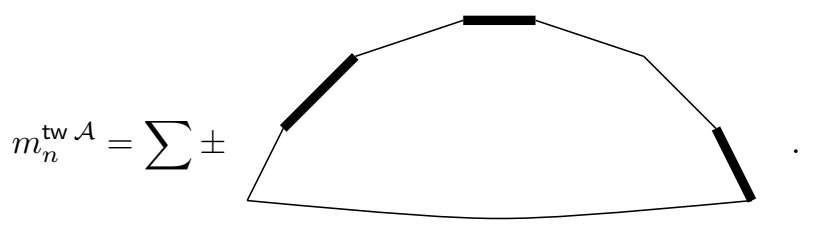

$\mathrm{m}$

Here the fat edges symbolize tensor powers of $\delta$ 's to be inserted in all possible places and in any number. The concatenation of the thin edges represents a given tensor $f_{n} \otimes f_{n-1} \otimes \cdots \otimes f_{1}$.

It is worthwile to make the formula explicit for $m_{1}$. If $f$ is a morphism of even degree from $(B, \delta)$ to $\left(B^{\prime}, \delta^{\prime}\right)$, we have

$$
\begin{aligned}
m_{1}^{\mathrm{tw} \mathcal{A}}(f)= & m_{1}(f)-m_{2}\left(\delta^{\prime}, f\right)+m_{2}(f, \delta) \\
& \quad-m_{3}\left(\delta^{\prime}, \delta^{\prime}, f\right)+m_{3}\left(\delta^{\prime}, f, \delta\right)-m_{3}(f, \delta, \delta) \\
+ & m_{4}\left(\delta^{\prime}, \delta^{\prime}, \delta^{\prime}, f\right)-m_{4}\left(\delta^{\prime}, \delta^{\prime}, f, \delta\right)+m_{4}\left(\delta^{\prime}, f, \delta, \delta\right)-m_{4}(f, \delta, \delta, \delta)+\ldots
\end{aligned}
$$

If $f$ is of odd degree, we have

$$
\begin{aligned}
m_{1}^{\mathrm{tw} \mathcal{A}}(f)= & m_{1}(f)-m_{2}\left(\delta^{\prime}, f\right)-m_{2}(f, \delta) \\
& \quad-m_{3}\left(\delta^{\prime}, \delta^{\prime}, f\right)-m_{3}\left(\delta^{\prime}, f, \delta\right)-m_{3}(f, \delta, \delta) \\
+ & m_{4}\left(\delta^{\prime}, \delta^{\prime}, \delta^{\prime}, f\right)+m_{4}\left(\delta^{\prime}, \delta^{\prime}, f, \delta\right)+m_{4}\left(\delta^{\prime}, f, \delta, \delta\right)+m_{4}(f, \delta, \delta, \delta)+\ldots
\end{aligned}
$$


In the sequel, we identify $\mathcal{A}$ with the subcategory of the objects $(A, 0)$ of $\mathbf{Z} \mathcal{A}$. With this convention, the functor $Y_{1}$ of (7.5) sends an object $A \in \mathcal{A}$ to $(A, \delta=0)$. The functor $Y_{2}$ sends $B=\left(A_{1}, \ldots, A_{n}\right)$ to $Y A_{1} \oplus \cdots \oplus Y A_{n}$ (where $Y$ denotes the canonical extension of the Yoneda functor to $\mathbf{Z} \mathcal{A}$ ) endowed with the multiplications

$$
m_{n}=\sum_{t=0}^{\infty}(-1)^{\frac{t(t-1)}{2}} m_{n+t} \circ\left(\delta^{\otimes t} \otimes \mathbf{1}^{\otimes n}\right), n \geqslant 1,
$$

where the $m_{n+t}$ on the right hand side denote the multiplications of the direct sum of the $Y A_{i}$.

\subsection{Description of filtered modules by twisted objects}

Let $B$ be an associative $k$-algebra with 1 , let $M_{1}, \ldots, M_{n}$ be $B$-modules and let filt $\left(M_{i}\right)$ denote the full subcategory of the category of right $B$-modules whose objects admit finite filtrations with subquotients among the $M_{i}$. Let $\mathcal{A}$ be the $A_{\infty}$-category with objects $1, \ldots, n$ and with morphism spaces

$$
\mathcal{A}(i, j)=\operatorname{Ext}_{B}^{*}\left(M_{i}, M_{j}\right) .
$$

The composition maps of $\mathcal{A}$ are defined as follows: The differential $m_{1}$ vanishes, the composition $m_{2}$ is the Yoneda composition, and the $m_{i}, i>3$, are the canonical higher compositions constructed as in $(3.3,3.4)$. We assume that these may be chosen so as to make $\mathcal{A}$ strictly unital. For example, this holds if $\operatorname{Ext}_{B}^{*}\left(M_{i}, M_{i}\right)$ is one-dimensional and $\operatorname{Ext}_{B}^{*}\left(M_{i}, M_{j}\right)=0$ for $i>j$ (cf. 3.5) or if the $M_{i}$ are the simple modules over a finite-dimensional basic algebra $B$. Indeed, in the latter case, both $B$ and the dg algebra computing the extensions are augmented over a product of fields (cf. 3.5).

Let filt $\mathcal{A}$ denote the full subcategory of $H^{0}$ tw $\mathcal{A}$ whose objects are the $(B, \delta)$ such that $B$ is a filtered direct sum of (non shifted) copies of objects of $\mathcal{A}$ (we use the alternative description of tw $\mathcal{A}$ and identify $\mathcal{A}$ with a subcategory of $\mathbf{Z} \mathcal{A}$ ).

Theorem. There is an equivalence of categories

$$
\text { filt } \mathcal{A} \stackrel{\sim}{\rightarrow} \text { filt }\left(M_{i}\right) .
$$

Although the category filt $\mathcal{A} \subset H^{0}$ tw $\mathcal{A}$ looks complicated, it is often quite easy to describe in practice. We illustrate this in two examples below.

The theorem is a consequence of (7.5) and (6.5). Indeed, by its construction, the category $\mathcal{A}$ is the $A_{\infty}$-Ext-category of the $B$-modules $M_{1}, \ldots, M_{n}$ in the sense of problem 2 of (2.1). So by (6.4) and (6.5), there is a triangle functor

$$
F: \mathcal{D}_{\infty} \mathcal{A} \rightarrow \mathcal{D} B
$$

inducing an equivalence

$$
\operatorname{tria}(Y 1, \ldots, Y n) \stackrel{\sim}{\rightarrow} \operatorname{tria}\left(M_{1}, \ldots, M_{n}\right) .
$$

By (7.5), the Yoneda functor induces an equivalence

$$
H^{0} \operatorname{tw} \mathcal{A} \rightarrow \operatorname{tria}(Y 1, \ldots, Y n) .
$$


Thus we get an equivalence

$$
H^{0} \operatorname{tw} \mathcal{A} \rightarrow \operatorname{tria}\left(M_{1}, \ldots, M_{n}\right)
$$

which takes the object $i$ to the corresponding module $M_{i}$ and therefore induces an equivalence

$$
\text { filt } \mathcal{A} \stackrel{\sim}{\rightarrow} \text { filt }\left(M_{i}\right) \text {. }
$$

\subsection{Example: Reconstruction from simple modules}

Let $B$ be a finite-dimensional basic algebra. Theorem 7.7 allows us in particular to reconstruct the category $\bmod B$ of finite-dimensional $B$-modules from the $A_{\infty}$ Ext-category of the simple $B$-modules.

We illustrate this by an example. Let $B$ be the algebra given by the quiver with relations

$$
x \stackrel{\alpha}{\rightarrow} y \stackrel{\beta}{\rightarrow} z \stackrel{\gamma}{\rightarrow} t, \gamma \beta \alpha=0
$$

and let $M_{1}=S_{x}, \ldots, M_{4}=S_{t}$ be the four simple $B$-modules. The $A_{\infty}$-category $\mathcal{A}$ of (7.7) is then given by the quiver with relations

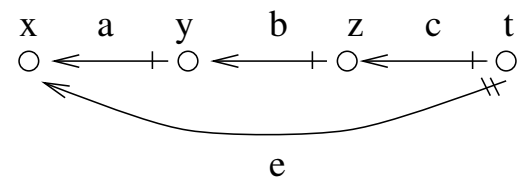

where the arrows $a, b, c$ are of degree 1 , the arrow $e$ is of degree 2, and all compositions other than $m_{3}(a, b, c)=e$ are defined to be zero (this example has already appeared in 3.3). We would like to determine the subcategory filt $\mathcal{A}$ of $H^{0}$ tw $\mathcal{A}$ whose objects are the $(A, \delta)$ where $A$ is a filtered sum of objects of $\mathcal{A}$ (we use the alternative description and identify $\mathcal{A}$ with a subcategory of $\mathbf{Z} \mathcal{A}$ ). By an obvious abuse of notation, we have

$$
A=V_{x} \otimes x \oplus \ldots \oplus V_{t} \otimes t
$$

where $V_{x}, \ldots, V_{t}$ are finite-dimensional vector spaces and the datum of

$$
\delta \in \operatorname{Hom}_{\mathbf{Z} \mathcal{A}}^{1}(A, A)
$$

corresponds to the datum of three linear maps $V_{a}, V_{b}, V_{c}$ :

$$
V_{x} \stackrel{V_{a}}{\longleftarrow} V_{y} \stackrel{V_{b}}{\longleftarrow} V_{z} \stackrel{V_{c}}{\longleftarrow} V_{t} \text {. }
$$

The condition

$$
m_{1}(\delta)+m_{2}(\delta, \delta)-m_{3}(\delta, \delta, \delta)-\ldots=0
$$

translates into $V_{a} V_{b} V_{c}=0$. By definition, morphisms from $(A, \delta)$ to $\left(A^{\prime}, \delta^{\prime}\right)$ are in bijection with

$$
H^{0} \operatorname{Hom}_{\mathrm{tw} \mathcal{A}}\left((A, \delta),\left(A^{\prime}, \delta^{\prime}\right)\right)
$$

Now $\operatorname{Hom}_{\mathcal{A}}^{-1}$ and hence

$$
\operatorname{Hom}_{\mathrm{tw} \mathcal{A}}^{-1}\left((A, \delta),\left(A^{\prime}, \delta^{\prime}\right)\right)
$$


vanishes so that the homology classes in degree zero are exactly the zero cycles. Now it is easy to see that the elements $f$ of $\operatorname{Hom}_{\mathrm{tw}} \mathcal{A}\left((A, \delta),\left(A^{\prime}, \delta^{\prime}\right)\right)$ correspond exactly to the quadruples of linear maps

$$
f_{x}: V_{x} \rightarrow V_{x}^{\prime}, \ldots, f_{t}: V_{t} \rightarrow V_{t}^{\prime}
$$

and the condition

$$
m_{1}(f)-m_{2}\left(\delta^{\prime}, f\right)+m_{2}(f, \delta)-m_{3}(\ldots)+\ldots=0
$$

translates into

$$
f_{x} V_{a}=V_{a}^{\prime} f_{y}, \ldots, f_{z} V_{c}=V_{c}^{\prime} f_{t} .
$$

Hence, as expected, the category filt $\mathcal{A}$ is equivalent to the category of contravariant representations of the quiver with relations

$$
x \stackrel{\alpha}{\rightarrow} y \stackrel{\beta}{\rightarrow} z \stackrel{\gamma}{\rightarrow} t, \gamma \beta \alpha=0,
$$

i.e. to the category $\bmod B$ of finite-dimensional right modules over the algebra $B$. This confirms theorem 7.7 in this example.

\subsection{Example: Classifying filtered modules}

This example arose from an email exchange with T. Brüstle. Consider the following quiver $Q$ of type $D_{4}$

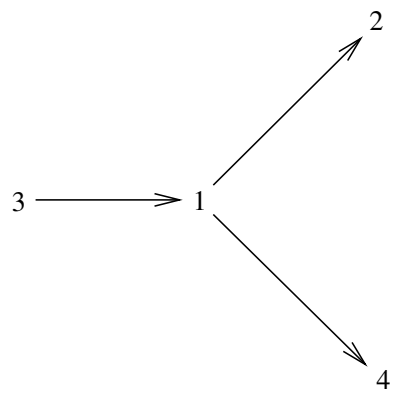

Denote by $S_{i}$ its simple (covariant) representation associated with the point $i, 1 \leqslant$ $i \leqslant 4$. Let $\Delta_{1}=S_{1}, \Delta_{2}=S_{2}, \Delta_{4}=S_{4}$ and let $\Delta_{3}$ be the following indecomposable representation

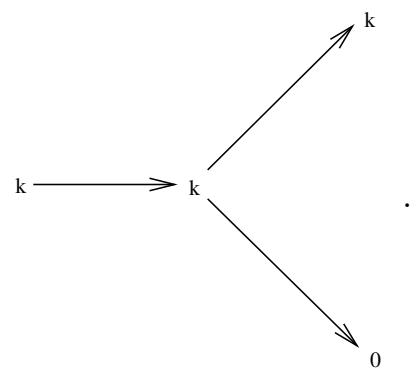

We are interested in the category filt $\left(\Delta_{1}, \ldots, \Delta_{4}\right)$ of representations of $Q$ admitting a filtration with subquotients among the $\Delta_{i}$. The $A_{\infty}$-Ext-category $\mathcal{A}$ of the $\Delta_{i}$ is 
given by the quiver

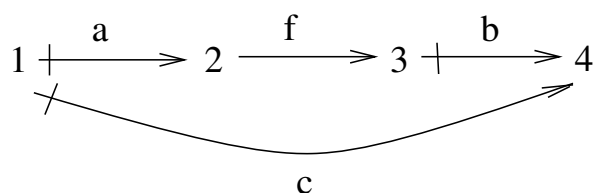

where $a, b, c$ are of degree $1, f$ is of degree 0 and all multiplications other than $m_{3}(b, f, a)=c$ are defined to be zero. As in example 7.8 , the subcategory filt $\mathcal{A}$ of $H^{0}$ tw $\mathcal{A}$ can be described as follows: Its objects are the finite-dimensional (covariant) representations $V$ of the quiver

$$
2 \stackrel{a}{\leftarrow} 1 \stackrel{c}{\rightarrow} 4 \stackrel{b}{\leftarrow} 3
$$

of type $A_{4}$. Its morphisms $f: V \rightarrow V^{\prime}$ are the quintuples of linear maps $f_{i}: V_{i} \rightarrow V_{i}^{\prime}$ and $\varphi: V_{2} \rightarrow V_{3}^{\prime}$

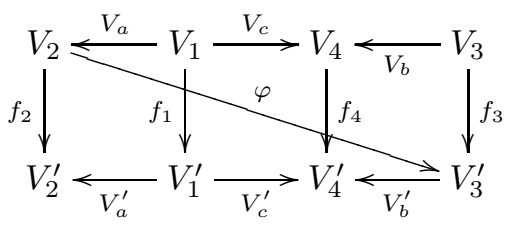

satisfying

$$
\begin{array}{r}
0=V_{a}^{\prime} f_{1}-f_{2} V_{a}, 0=V_{b}^{\prime} f_{3}-f_{4} V_{b} \\
0=V_{c}^{\prime} f_{1}-f_{4} V_{c}+V_{b}^{\prime} \varphi V_{a} \quad(*)
\end{array}
$$

Thanks to theorem 7.7 , we have an equivalence

$$
\text { filt } \mathcal{A} \stackrel{\sim}{\rightarrow} \text { filt }\left(\Delta_{1}, \ldots, \Delta_{4}\right) .
$$

The term $V_{b}^{\prime} \varphi V_{a}$ in $(*)$ is essential: If it is omitted, the problem is that of classifying the representations of the quiver of type $A_{4}$ above. Then we find 10 isoclasses of indecomposables instead of the 9 isoclasses present on the right hand side.

\section{On the proofs}

\subsection{The minimality theorem 3.3}

The article [13] gives a detailed account of various approaches to this theorem (for the case of connected $\mathrm{dg}$ algebras $A$ ). For the case where $A$ is a $\mathbf{Z}$-graded $\operatorname{dg}$ algebra, it is proved in [32]. Here one also finds new explicit formulas for the higher multiplication maps on $H^{*} A$.

In the general case, one may proceed as follows [28]: Let $A$ and $B$ be two $A_{\infty^{-}}$ algebras and $\left(f_{1}, \ldots, f_{s}\right)$ an $A_{s}$-morphism (i.e. the defining equation from 3.4 holds for $n \leqslant s)$. One can then define a morphism of complexes $c=c\left(f_{1}, \ldots, f_{s}\right)$ : $A^{\otimes s+1} \rightarrow B$ such that $\left(f_{1}, \ldots, f_{s}\right)$ extends to an $A_{s+1}$-morphism $\left(f_{1}, \ldots, f_{s+1}\right)$ iff $c$ is null homotopic and in this case, the homotopy is given by $f_{s+1}$. Here, $A$ and $B$ are endowed with the differentials given by $m_{1}$. It follows that if $\left(B, m_{1}\right)$ is contractible, each $A_{s}$-morphism with target $B$ extends to an $A_{\infty}$-morphism. 
Now suppose that $A$ is an $A_{\infty}$-algebra and $A=A^{\prime} \oplus N$ a decomposition of the complex $\left(A, m_{1}\right)$ with contractible $N$. We wish to make $A^{\prime}$ into an $A_{\infty}$-algebra quasi-isomorphic to $A$. For this, we make $N$ into an $A_{\infty}$-algebra by taking all $m_{n}$, $n \geqslant 2$, to vanish. Since $N$ is contractible, the morphism of complexes $A \rightarrow N$ extends to an $A_{\infty}$-morphism. We thus obtain a dg coalgebra morphism $p: \bar{T} S A \rightarrow \bar{T} S N$ (cf. 3.6). It is easy to check that its kernel in the category of graded coalgebras is isomorphic to $\bar{T} S A^{\prime}$. Thanks to 3.6 , this yields an $A_{\infty}$-algebra structure on $A^{\prime}$ and an $A_{\infty}$-morphism $A^{\prime} \rightarrow A$ extending the inclusion.

It is easy to see that an $A_{\infty}$-morphism $f$ between minimal $A_{\infty}$-algebras is invertible iff $f_{1}$ is invertible. This yields the uniqueness assertion of the theorem.

\subsection{The theorem on homotopies 3.7}

The theorem results from the facts, proved in $[\mathbf{2 8}]$, that

1) the bar construction functor (3.6) is an equivalence from the category of $A_{\infty^{-}}$ algebras onto the full subcategory of fibrant-cofibrant objects of a closed model category of coalgebras and

2) the intrinsic homotopy relation of this closed model category induces the explicit relation given in 3.7.

Alternatively, one can prove b) by the following argument taken from $[\mathbf{2 4}, 4.5]$ : One shows that the quasi-isomorphism $i_{A}: H^{*} A \rightarrow A$ constructed above is actually an homotopy equivalence. The given morphism $s: A \rightarrow B$ thus fits into a diagram

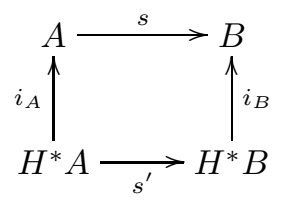

of $A_{\infty}$-algebras which is commutative up to homotopy. The first component $s_{1}^{\prime}$ identifies with $H^{*} s$. So $H^{*} s$ is invertible iff $s^{\prime}$ is an isomorhism iff $s$ is a homotopy equivalence.

\section{3. $\quad A_{\infty}$-modules (section 4 )}

Theorem 4.2 on $A_{\infty}$-modules has an analogous (but simpler) proof as the corresponding theorem 3.7 on $A_{\infty}$-algebras, cf. [28].

Part a) of theorem 6.5 is proved in [22, section 7]. The existence of the minimal model in b) is shown in [12]. Uniqueness is easy (as in the case of $A_{\infty}$-algebras).

\subsection{Triangulated structure (section 5)}

The following argument is taken from [28]: The category of $A_{\infty}$-modules over an $A_{\infty}$-algebra $A$ is equivalent to a full subcategory $\mathcal{C}^{\prime}$ of the category $\mathcal{C}$ of differential counital comodules over the counital dg coalgebra TSA (cf. 4.2). We endow the category $\mathcal{C}$ with the class of exact sequences whose underlying sequences of graded comodules split. As in $[\mathbf{2 1}, 2.2]$, one shows that $\mathcal{C}$ is a Frobenius category. The associated stable category is thus triangulated. This category equals the homotopy category $\mathcal{H}$ of the category of $\mathrm{dg}$ counital comodules. It is easy to check that the 
essential image $\mathcal{H}^{\prime}$ of $\mathcal{C}^{\prime}$ in $\mathcal{H}$ is a triangulated subcategory. By theorem 4.2, $\mathcal{H}^{\prime}$ is equivalent to the derived category $\mathcal{D}_{\infty} A$. Exact sequences of strict morphisms of $A_{\infty}$-modules give rise to exact sequences of coinduced graded $T S A$-comodules. Such sequences split in the graded category (coinduced modules are injective). Thus each exact sequence of strict morphisms of $A_{\infty}$-modules gives rise to a canonical triangle. When we have an arbitrary triangle $(K, L, M)$ of $\mathcal{H}^{\prime}$, we can form a mapping cylinder over the morphism $M \rightarrow S K$ to obtain an isomorphic triangle $K \rightarrow L^{\prime} \rightarrow M \rightarrow S K$. By the construction of the mapping cylinder, the sequence of graded comodules $K \rightarrow L^{\prime} \rightarrow M$ identifies with the canonical sequence $K \rightarrow K \oplus M \rightarrow M$. So the sequence $K \rightarrow L^{\prime} \rightarrow M$ does come from a sequence of strict $A_{\infty}$-morphisms.

\subsection{Standard functors (section 6)}

The assertions in this section are based on the following description [28] of the derived category $\mathcal{D}_{\infty} A$ : The functor $M \mapsto M \otimes T S A$ is an equivalence of the category of $A_{\infty}$-modules onto the full subcategory of fibrant-cofibrant objects of a closed model category of counital dg comodules over TSA. Thus it induces an equivalence of $\mathcal{D}_{\infty} A$ onto the corresponding homotopy category.

\subsection{Twisted objects (section 7)}

We follow [28]. Below, we will prove that tw $\mathcal{A}$ is an $A_{\infty}$-category. The proof that $Y_{2}$ is an $A_{\infty}$-functor follows the same lines. It remains to be proved that $Y_{2}$ induces an equivalence of $H^{0}$ tw $\mathcal{A}$ onto its image in the homotopy category (=derived category) of $A_{\infty}$-modules. For this, let $B_{1}$ and $B_{2}$ be two twisted objects and consider the map

$$
H^{*} \operatorname{tw} \mathcal{A}\left(B_{1}, B_{2}\right) \rightarrow H^{*} \operatorname{Hom}_{\mathcal{A}}\left(Y_{2} B_{1}, Y_{2} B_{2}\right)
$$

induced by $Y_{2}$. We have to show it is an isomorphism. Using the canonical filtrations of $B_{1}$ and $B_{2}$, one easily reduces this to the special case where the $B_{i}$ are objects $A_{i}$ of $\mathcal{A}$ (and thus the corresponding twisting elements $\delta$ vanish). Then one has to show that the map

$$
H^{*} \mathcal{A}\left(A_{1}, A_{2}\right) \rightarrow H^{*} \operatorname{Hom}_{\mathcal{A}}\left(Y A_{1}, Y A_{2}\right)
$$

is bijective. Now using the strict identity of $A_{1}$, it is not hard to construct an explicit inverse of this map.

Let us show that tw $\mathcal{A}$ is an $A_{\infty}$-category. Let $\mathcal{B}$ be the datum of

- the same class of objects as tw $\mathcal{A}$,

- the same graded morphism spaces as tw $\mathcal{A}$,

- the natural composition maps $m_{n}^{\prime}$ obtained by extending the maps $m_{n}^{\mathbf{Z} \mathcal{A}}$ to matrices.

It is clear that $\mathcal{B}$ is an $A_{\infty}$-category. For each sequence $B_{0}, \ldots, B_{n}$ of objects of $\mathcal{B}$, let $b_{n}^{\prime}$ be the map

$$
S\left(B_{n-1}, B_{n}\right) \otimes S\left(B_{n-2}, B_{n-1}\right) \otimes \ldots \otimes S\left(B_{0}, B_{1}\right) \rightarrow S\left(B_{0}, B_{n}\right)
$$

deduced from $m_{n}^{\prime}$ as in the bar construction (3.6). Here, $S\left(B_{i-1}, B_{i}\right)$ denotes the suspension of the morphism space $\left(B_{i-1}, B_{i}\right)$. For each $0 \leqslant i \leqslant n$, let $\alpha: k \rightarrow$ 
$S\left(B_{i}, B_{i}\right)$ be the composition of the degree 1 map

$$
k \rightarrow\left(B_{i}, B_{i}\right), 1 \mapsto \delta
$$

with the canonical morphism $s:\left(B_{i}, B_{i}\right) \rightarrow S\left(B_{i}, B_{i}\right)$ of degree -1 . Note that $\alpha$ is of degree 0 . A sign check shows that the identity 7.1 translates into

$$
0=\sum_{i \geqslant 1} b_{i}^{\prime} \circ \alpha^{\otimes i} .
$$

Now for each $t \geqslant 0$, let $\varphi_{n, t}$ be the sum of the maps

$$
\mathbf{1}^{\otimes i_{1}} \otimes \alpha^{\otimes j_{1}} \otimes \ldots \otimes \mathbf{1}^{\otimes i_{r}} \otimes \alpha^{\otimes j_{r}}
$$

defined on

$$
S\left(B_{n-1}, B_{n}\right) \otimes \ldots \otimes S\left(B_{0}, B_{1}\right)
$$

where $i_{1}+\cdots+i_{r}=n$ and $j_{1}+\cdots+j_{r}=t$ as in 7.6. Note that for each decomposition $n=n_{1}+n_{2}$, we have

$$
\varphi_{n, t}=\sum_{t=t_{1}+t_{2}} \varphi_{n_{1}, t_{1}} \otimes \varphi_{n_{2}, t_{2}} .
$$

Define

$$
b_{n}=\sum_{t \geqslant 0} b_{n+t}^{\prime} \circ \varphi_{n, t} .
$$

A sign check shows that $b_{n}$ corresponds to the twisted composition $m_{n}^{\text {tw } \mathcal{A}}$ defined in 7.6. We have to show that

$$
\sum b_{l}\left(\mathbf{1}^{\otimes i} \otimes b_{j} \otimes \mathbf{1}^{\otimes k^{\prime}}\right)=0,
$$

where the sum runs over the decompositions $n=i+j+k^{\prime}$ and we put $l=i+1+k^{\prime}$. Now we have

$$
\begin{aligned}
\sum b_{l}\left(\mathbf{1}^{\otimes i} \otimes b_{j} \otimes \mathbf{1}^{\otimes k^{\prime}}\right) & =\sum b_{l+s}^{\prime} \varphi_{l, s}\left(\mathbf{1}^{\otimes i} \otimes\left(b_{j+t}^{\prime} \varphi_{j, t}\right) \otimes \mathbf{1}^{\otimes k^{\prime}}\right) \\
& =\sum b_{l+s}^{\prime}\left(\varphi_{i, s_{1}} \otimes\left(b_{j+t}^{\prime} \varphi_{j, t}\right) \otimes \varphi_{k^{\prime}, s_{2}}\right)
\end{aligned}
$$

the indices run trough the integers $\geqslant 0$ such that $s=s_{1}+s_{2}$. Here, we have used the decomposition formula 8.1 and the fact that $\alpha$ is of degree 0 . Let $\Sigma_{1}$ denote the last sum. Graphically, the terms of $\Sigma_{1}$ correspond to diagrams

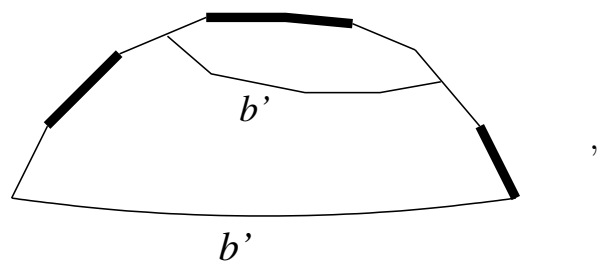

where the fat edges symbolize $\alpha$ 's to be inserted in any number and in all positions such that the arc above the upper $b^{\prime}$ is not entirely fat (it must always contain a 
thin piece symbolizing the $j \geqslant 1$ arguments of $b_{j}$ ). To show that $\Sigma_{1}$ vanishes, we compare it to

$$
\sum_{r \geqslant 0} \sum b_{L}^{\prime}\left(\mathbf{1}^{\otimes I} \otimes b_{J}^{\prime} \otimes, \mathbf{1}^{\otimes K}\right) \varphi_{n, r}
$$

where the inner sum runs over all decompositions of $N=n+r$ into $N=I+J+K$ and we put $L=I+1+K$. Since $\mathcal{B}$ is an $A_{\infty}$-category, the inner sum vanishes for each $r$. On the other hand, the whole expression equals

$$
\sum b_{L}^{\prime}\left(\varphi_{I^{\prime}, r_{1}} \otimes\left(b_{J^{\prime}+r_{2}}^{\prime} \varphi_{J^{\prime}, r_{2}}\right) \otimes \varphi_{K^{\prime}, r_{3}}\right),
$$

where the sum runs over the non negative integers $r, r_{1}, r_{2}, r_{3}, I^{\prime}, J^{\prime}, K^{\prime}$ satisfying $r=r_{1}+r_{2}+r_{3}, I^{\prime}+J^{\prime}+K^{\prime}=n$, and we put $L=\left(I^{\prime}+r_{1}\right)+1+\left(K^{\prime}+r_{3}\right)$. Let us denote the last sum by $\Sigma_{2}$. Each term of $\Sigma_{1}$ appears exactly once in $\Sigma_{2}$ but not all terms of $\Sigma_{2}$ appear in $\Sigma_{1}$. Those that do not appear are precisely the terms where $J^{\prime}=0$, i.e. the terms

$$
b_{L}^{\prime}\left(\varphi_{I^{\prime}, r_{1}} \otimes\left(b_{r_{2}}^{\prime} \varphi_{0, r_{2}}\right) \otimes \varphi_{K^{\prime}, r_{3}}\right) .
$$

Graphically, they correspond to diagrams

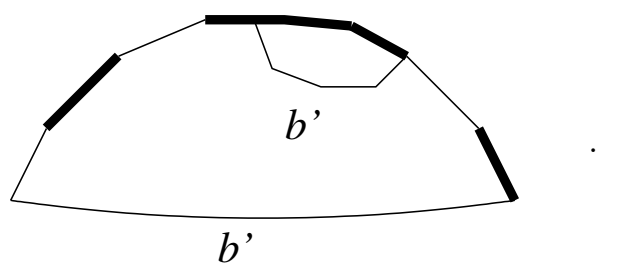

Now we have $b_{r_{2}}^{\prime} \varphi_{0, r_{2}}=b_{r_{2}}^{\prime} \circ \alpha^{\otimes r_{2}}$ so that the sum over all $r_{2} \geqslant 0$ of these terms vanishes. Thus we have $\Sigma_{1}=\Sigma_{2}=0$.

\section{References}

[1] J. F. Adams, Infinite loop spaces, Ann. of Math. Stud., Vol. 90, Princeton Univ. Press, Princeton, N.J., 1978.

[2] M. Auslander, I. Reiten, S. Smalø, Representation theory of Artin algebras, Cambridge Studies in Adv. Math., Vol. 36, Cambridge University Press, 1995.

[3] J. M. Boardman, R. M. Vogt, Homotopy Invariant Algebraic Structures on Topological Spaces, Lecture Notes in Mathematics 347, Springer, 1973.

[4] M. Bökstedt, A. Neeman, Homotopy limits in triangulated categories, Compositio Math. 86 (1993), 209-234.

[5] A. I. Bondal, M. M. Kapranov, Enhanced triangulated categories (Russian) Mat. Sb. 181 (1990), 669-683. Translated in Math. USSR, Sb. 70, (1991), 93-107.

[6] H. Cartan, S. Eilenberg, Homological Algebra, Princeton University Press, 1956. 
[7] P. Deligne, P. Griffiths, J. Morgan, D. Sullivan, Real Homotopy Theory of Kähler Manifols, Invent. math. 29, 1975, 245-274.

[8] K. Fukaya, Morse homotopy, $A^{\infty}$-category and Floer homologies, MSRI preprint No. 020-94 (1993).

[9] P. Gabriel, A. V. Roiter, Representations of finite-dimensional algebras, Algebra VIII, Encyclopaedia Math. Sci., Vol. 73, Springer Verlag, 1992.

[10] S. I. Gelfand, Yu. I. Manin, Methods of homological algebra, Transl. from the Russian, Springer, Berlin, 1996.

[11] E. Getzler, J. D. S. Jones, $A_{\infty}$-algebras and the cyclic bar complex, Illinois J. Math. 34 (1990), 256-283.

[12] V. K. A. M. Gugenheim, L. A. Lambe, J. D. Stasheff, Perturbation theory in differential homological algebra II, Illinois J. Math. 35 (1991), 357-373.

[13] L. Johansson, L. Lambe, Transferring Algebra Structures Up to Homology Equivalence, Preprint, 1996, to appear in Math. Scand.

[14] T. V. Kadeishvili, On the theory of homology of fiber spaces (Russian), Uspekhi Mat. Nauk 35 (1980), 183-188. Translated in Russ. Math. Surv. 35 (1980), 231-238.

[15] T. V. Kadeishvili, The algebraic structure in the homology of an $A(\infty)$-algebra (Russian), Soobshch. Akad. Nauk Gruzin. SSR 108 (1982), 249-252.

[16] T. V. Kadeishvili, The category of differential coalgebras and the category of $A(\infty)$-algebras (Russian), Trudy Tbiliss. Mat. Inst. Razmadze Akad. Nauk Gruzin. SSR 77 (1985), 50-70.

[17] T. V. Kadeishvili, Twisted tensor products for the category of $A(\infty)$-algebras and $A(\infty)$-modules (Russian, English summary), Trudy Tbiliss. Mat. Inst. Razmadze Akad. Nauk Gruzin. SSR 83 (1986), 26-45.

[18] T. V. Kadeishvili, The functor $D$ for a category of $A(\infty)$-algebras (Russian), Soobshch. Akad. Nauk Gruzin. SSR 125 (1987), 273-276.

[19] T. V. Kadeishvili, The structure of the $A(\infty)$-algebra, and the Hochschild and Harrison cohomologies (Russian), Trudy Tbiliss. Mat. Inst. Razmadze Akad. Nauk Gruzin. SSR 91 (1988), 19-27.

[20] M. Kashiwara, P. Schapira, Sheaves on Manifolds, Grundlehren 292, SpringerVerlag, Berlin Heidelberg, 1990.

[21] B. Keller, Deriving DG categories, Ann. Scient. Ec. Norm. Sup. 27 (1994), 63-102.

[22] B. Keller, Bimodule complexes via strong homotopy actions, Preprint, 1999, 15 pages. Available at http://www.math.jussieu.fr/ keller/recent.html

[23] M. Kontsevich, Homological algebra of Mirror Symmetry, Proceedings of the International Congress of Mathematicians, Zürich, Switzerland 1994, Vol. 1, Birkhäuser Basel, 1995, 120-139.

[24] M. Kontsevich, Deformation Quantization of Poisson manifolds, I, available at http://xxx.lanl.gov/abs/q-alg/9709040. 
[25] M. Kontsevich, Triangulated categories and geometry, Course at the Ecole Normale Supérieure, Paris, March and April 1998.

[26] M. Kontsevich, Y. Soibelman, Deformations of algebras over operads and Deligne's conjecture, available at http://xxx.lanl.gov/abs/math.QA/0001151.

[27] M. Kontsevich, Y. Soibelman, Homological mirror symmetry and torus fibrations, available at http://xxx.lanl.gov/abs/math.SG/0011041.

[28] K. Lefèvre, On $A_{\infty}$-categories, in preparation.

[29] M. Markl, Simplex, Associahedron, and Cyclohedron, Contemp. Math. 227 (1999), 235-265.

[30] J. P. May, The Geometry of Iterated Loop Spaces, Lecture Notes in Mathematics 271, Springer, 1972.

[31] J. McCleary (Ed.), Higher homotopy structures in topology and mathematical physics, Contemp. Math., 227, Amer. Math. Soc., Providence, RI, 1999.

[32] S. A. Merkulov, Strong homotopy algebras of a Kähler manifold, available at http://xxx.lanl.gov/abs/math. AG/9809172.

[33] B. Mitchell, Rings with several objects, Adv. in Math. 8 (1972), 1-161.

[34] S. Ovsienko, On derived categories of representations categories, in: "XVIII Allunion algebraic conference", Proceedings, Part II, Kishinev, (1985), 71.

[35] S. Ovsienko, Bimodule and matrix problems, Preprint, 1998.

[36] M. Penkava, A. Schwarz, $A_{\infty}$ Algebras and the Cohomology of Moduli Spaces, in: Gindikin, S. G. (ed.) et al., Lie groups and Lie algebras: E. B. Dynkin's seminar. American Mathematical Society. Transl., Ser. 2, Am. Math. Soc. 169, 91-107 (1995), also available at http://xxx.lanl.gov/abs/hep-th/9408064

[37] A. Polishchuk, E. Zaslow, Categorical Mirror Symmetry: The Elliptic Curve, http://xxx.lanl.gov/abs/math/9801119.

[38] A. Prouté, Algèbres différentielles fortement homotopiquement associatives, Thèse d'Etat, Université Paris VII, 1984.

[39] J. Rickard, Morita theory for derived categories, J. London Math. Soc. (2) 39 (1989), 436-456.

[40] C. M. Ringel, Tame algebras and integral quadratic forms, Lecture Notes in Mathematics, Vol. 1099, Springer Verlag, 1984.

[41] P. Seidel, R. Thomas, Braid group actions on derived categories of coherent sheaves, to appear in Duke Math. J., http://xxx.lanl.gov/abs/math.AG/0001043

[42] E. Sköldberg, (Co)homology of monomial algebras, Ph. D. Thesis, Stockholm University, 1997.

[43] V. A. Smirnov, Homology of fiber spaces (Russian), Uspekhi Mat. Nauk 35 (1980), 227-230. Translated in Russ. Math. Surveys 35 (1980), 294-298.

[44] N. Spaltenstein, Resolutions of unbounded complexes, Compositio Math. 65 (1988), 121-154. 
[45] J. D. Stasheff, Homotopy associativity of H-spaces, I, Trans. Amer. Math. Soc. 108 (1963), 275-292.

[46] J. D. Stasheff, Homotopy associativity of H-spaces, II, Trans. Amer. Math. Soc. 108 (1963), 293-312.

[47] J. D. Stasheff, $H$-spaces from a homotopy point of view, Lecture notes in Mathematics 161, Springer, 1970.

[48] J. D. Stasheff, Differential graded Lie algebras, Quasi-Hopf algebras, and higher homotopy algebras, Quantum Groups, Proc. workshops, Euler Int. Math. Inst., Leningrad 1990, Lecture Notes in Mathematics 1510, Springer 1992, 120-137.

[49] J.-L. Verdier, Catégories dérivées, état 0, SGA 4 1/2, Springer LNM 569 (1977), 262-311.

[50] J.-L. Verdier, Des catégories dérivées des catégories abéliennes, Astérisque 239, Soc. Math. France, 1997.

[51] C. A. Weibel, Introduction to homological algebra, Cambridge Studies in Advanced Mathematics 38, Cambridge University Press, 1994.

[52] M. Wodzicki, Excision in cyclic homology and in rational algebraic $K$-theory, Ann. of Math. 129 (1989), 591-639.

This article may be accessed via WWW at http://www.rmi.acnet.ge/hha/ or by anonymous ftp at

ftp://ftp.rmi.acnet.ge/pub/hha/volumes/2001/n1/n1.(dvi,ps,dvi.gz,ps.gz)

Bernhard Keller keller@math.jussieu.fr

$$
\text { www.math.jussieu.fr/ } \sim \text { keller }
$$

UFR de Mathématiques

UMR 7586 du CNRS

Case 7012

Université Paris 7

2, place Jussieu

75251 Paris Cedex 05

France 